\title{
Light singlino dark matter at the LHC
}

\author{
Monoranjan Guchait ${ }^{*}$ and Arnab Roy ${ }^{\dagger}$ \\ Department of High Energy Physics, Tata Institute of Fundamental Research, \\ Homi Bhabha Road, Mumbai-400005, India
}

(Received 1 June 2020; accepted 25 September 2020; published 19 October 2020)

\begin{abstract}
The light singlinolike neutralino is expected to be a promising candidate for dark matter (DM) in the allowed parameter space of the next-to-minimal supersymmetric standard model. The DM annihilation process takes place via the light Higgs bosons which are natural in this model. Identifying the allowed region of parameter space including various constraints, the detection prospect of such light DM candidate and Higgs bosons are explored at the LHC with its high luminosity options. Light Higgs bosons and the DM candidate, the lightest singlinolike neutralino are indirectly produced at the LHC via the SM Higgs production and its subsequent decays. Jet substructure techniques are used to tag boosted Higgs. It is found that the favorable range of masses of Higgs bosons and neutralino, compatible with a low mass DM solution, can be discovered with a reasonable signal significance $(\sim 5 \sigma)$ at the LHC, with the center of mass energy $\sqrt{s}=14 \mathrm{TeV}$ and integrated luminosity options $\mathcal{L}=300 \mathrm{fb}^{-1}$ and $3000 \mathrm{fb}^{-1}$.
\end{abstract}

DOI: 10.1103/PhysRevD.102.075023

\section{INTRODUCTION}

Understanding the nature of dark matter (DM) candidate is of great interest in the present day of particle physics, particularly, in the context of beyond standard model (BSM) physics. Huge efforts are in place by various experiments to search for DM candidate via direct and indirect manner[1,2]. Unfortunately, still the candidate of DM remains elusive. Very recent observations from the PLANCK [3] experiment predict the limits of relic density at $2 \sigma$ as,

$$
\Omega h^{2}=0.12 \pm 0.001
$$

It is observed that the DM annihilation cross section at the weak scale naturally predicts relic density consistent with this PLANCK data. Currently, searches for DM candidates are one of the most exciting and challenging programs. Numerous dedicated experiments including the Large Hadron Collider (LHC) are involved in this endeavor, and have made considerable progress. However, all negative results in direct searches of DM experiments, lead to stringent limits on DM-nucleon scattering cross sections in terms of DM particle masses [4-9]. As we know, because of

\footnotetext{
"guchait@tifr.res.in

arnab.roy@tifr.res.in
}

Published by the American Physical Society under the terms of the Creative Commons Attribution 4.0 International license. Further distribution of this work must maintain attribution to the author(s) and the published article's title, journal citation, and DOI. Funded by SCOAP. the nonrelativistic nature of DM candidate, the DMnucleon scattering cross section can be separated into two parts, spin-independent(SI) and spin-dependent(SD). The SI part is mediated by scalars and increases with the mass of the nucleon, whereas the SD process involving axial-vector coupling with nuclear spin is mediated by gauge bosons. Obviously, the SD cross section is larger than SI because of the suppressed coupling due to light quark masses [10-12]. Recent measurements by the XENON1T experiment reported an upper limit of the DM-nucleon SI elastic scattering cross section at $4.1 \times$ $10^{-47} \mathrm{~cm}^{2}$ and $2 \times 10^{-44} \mathrm{~cm}^{2}$ corresponding to DM particle masses of $30 \mathrm{GeV}$ and $6 \mathrm{GeV}$, respectively [4]. These are the most stringent limits to date, whereas limits from LUX [5] and PANDA [6] are not competitive. With the detector upgrade in XENONnT experiment, the sensitivity is expected to improve by an order of magnitude [4]. It is to be noted that the XENON1T experiment is not sensitive to the lower range $(<6 \mathrm{GeV})$ of $\mathrm{DM}$ particle masses. However, there are few other experiments that are sensitive to this lower mass range of DM [7,13-15]. For instance, DarkSide-50 experiment searches for DM candidate covering the mass range $\lesssim 20 \mathrm{GeV}$, and lack of observation of any signal event leads to an exclusion limit on DM-nucleon SI cross section at $10^{-41} \mathrm{~cm}^{2}$, at $90 \%$ C.L, corresponding to the DM particle of mass $1.8 \mathrm{GeV}$ [7]. Similarly, the SD DM-proton and DM-neutron scattering cross sections are also constrained for a reasonably wide range of DM particle masses. The exclusion limit on SD DM-neutron scattering cross section also come from XENON1T, which predicts at $90 \% \mathrm{CL}$, an upper limit $6.3 \times 10^{-42} \mathrm{~cm}^{2}$ for $30 \mathrm{GeV} \mathrm{DM}$ particle mass, and it increases further to $3 \times 10^{-39} \mathrm{~cm}^{2}$ for 
$6 \mathrm{GeV}$ mass [8]. The most stringent SD cross section limit to date on DM-proton scattering cross section at $90 \%$ C.L is $\sim 3 \times 10^{-41} \mathrm{~cm}^{2}$ for $20 \mathrm{GeV}$ DM particle mass which comes from the PICO-60 [9] experiment. Apart from these direct searches, DM candidates are also explored indirectly at the LHC experiment. The DM particle produced in proton-proton collision at the LHC leaves an imbalance of missing energy signature in the detector because of its extremely weak interaction with matter. Hence, the final state consisting of a hard missing energy along with a recoil of visible energy is assumed to be a classical signature of DM. Currently, in both CMS and ATLAS experiments, searching for the signature of DM candidates are treated as a high priority analysis [16]. However, from the nonobservation of any signal events in data, model-dependent limits of DM particle masses are set by both CMS and ATLAS experiments $[17,18]$. Evidently, even in the presence of stringent constraints on DM particle masses from all direct and indirect searches, as discussed above, still a considerable range of lower $(\sim$ few $\mathrm{GeV})$ and higher $(\mathcal{O}(100) \mathrm{GeV})$ range of masses are not ruled out. Naturally, this observation attracts special attention to look for models, which can offer viable DM candidates of those mass ranges compatible with data. Motivated by this fact, in this paper we try to find models of DM particle corresponding to this lower range of masses, which can provide solution consistent with all constraints due to direct and indirect searches, as discussed above [4-9,17,18].

Variety of well-motivated BSM predicts a plethora of cold and warm DM particle candidates [19,20]. Among them, the most widely studied DM model is offered by minimal supersymmetric standard model (MSSM), where the most popular candidate for DM with conserved R-parity is the lightest neutralino $\left(\tilde{\chi}_{1}^{0}\right)$, a majorana spin $1 / 2$ particle. In order to obtain right relic density [Eq. (1.1)], the DM candidate is favored to be the Higgsino-like, and of the mass range $\sim 1 \mathrm{TeV}$ [21-23]. Interestingly, assuming neutralino as a thermal relic, the relic density bound sets a lower limit on the neutralino mass, $m_{\tilde{\chi}_{1}^{0}} \gtrsim 34 \mathrm{GeV}$, in the framework of MSSM [24]. Naturally, it rules out the possibility of having any DM candidate of very low mass ( $\sim$ few GeV) in MSSM. Like the MSSM, the theory of next-to-minimal supersymmetric standard model (NMSSM) [25-28] offers the lightest neutralino as a potential DM candidate. In the NMSSM, the Higgsino mass term $(\mu)$ is generated dynamically, in order to cure the $\mu$-problem [29], by adding a singlet scalar field with two Higgs doublet and extending the Higgs sector resulting in seven Higgs bosons states. Because of the interplay between model parameters in the Higgs sector, one or two of the Higgs boson states can be very light, even less than the mass of the SM-like Higgs boson, without violating any collider constraints[30-36]. Furthermore, the singlino, fermionic superpartner of singlet field, extends the neutralino sector with five physical states, where the lightest neutralino state plays the role as a DM candidate. In particular, even with a very low mass ( $\sim$ few $\mathrm{GeV})$, the neutralino, favorably to be singlinolike, appears as a viable dark matter candidate without violating any existing constraints predicted by several DM experiments [37-45]. In such a scenario, the possible DM annihilation process occurs via light Higgs bosons reproducing right cross section consistent with the relic density given by Eq. (1.1). This phenomena resembles the scenario of Higgs portal model, where light Higgs boson acts as a portal between the SM and non-SM sector [46]. Moreover, the DM(singlino)-nucleon scattering cross sections, both SI and SD, satisfy experimental limits predicted by direct searches, thanks to the presence of appropriate singlino composition in the lightest neutralino state. In this regard, naturally, the immediate and pertinent question to ask is about the prospect of detecting the signal of this low mass singlinolike DM candidate at the LHC. In literature, quite a few studies exist in this context predicting the discovery potential of DM candidate at the LHC $[43,44,47-50]$. The objective of this present study is to revisit this DM solution in the framework of NMSSM, and then explore the detection prospect of such a scenario at the LHC for the current and future luminosity options. More precisely, our goal is to find discovery potential of light singlinolike neutralino and Higgs boson states at the LHC, which in combination provide a right DM solution.

At the LHC, the direct production of light singlino state and singletlike Higgs bosons, having negligible coupling with fermions and gauge bosons are very much suppressed. In such a scenario, these particles can be produced indirectly via the production of some other intermediate particles which are having non-negligible couplings with those states $[36,44,51,52]$. For example, in this paper, we consider the production of non-SM-like light Higgs bosons via the decays of SM Higgs boson which is produced through a standard mechanism. Subsequently, light singlino states are produced via the decay of light non-SM-like Higgs bosons. It is to be noted that the corresponding branching ratios (BR) of all these decay modes are very much sensitive to model parameters, which will be discussed in detail in later sections.

The SM Higgs boson is considered to be produced via gluon-gluon fusion, which is the dominant production mechanism $[53,54]$. In order to give a boost to the final state, the SM Higgs boson is produced exclusively along with a jet. Consequently, the pair of lighter Higgs boson states originating from the decay of SM Higgs boson of mass $125 \mathrm{GeV}$ are moderately boosted $\left(p_{T} \sim 30-40 \mathrm{GeV}\right)$ with a reasonable separation between them, and so the decay products from those states emerge as a collimated object. Thus the jets coming from light Higgs decay appear as a single fat jet. Using jet substructure technique this "Higgs jet" (HJ) is tagged where the two subjets are likely to be $b$-like $[55,56]$. In summary, we focus on the signal final state consisting an $\mathrm{HJ}$ and missing energy, along with at least one untagged QCD jet. Considering this signal final 
state, we perform detail signal and SM background simulation and predict signal sensitivity for $300 \mathrm{fb}^{-1}$ and $3000 \mathrm{fb}^{-1}$ luminosity options.

The paper is organized as follows. In Sec. II, we review the NMSSM model briefly and discussed the region of parameter space compatible with relic density and DMnucleon scattering constraints as well. The relevant range of parameters are identified through a numerical scan. Signal and background simulation is presented in Sec. III, followed by a discussion on the results. Finally, the summary is presented in Sec. V.

\section{THE NMSSM MODEL AND DARK MATTER RELIC DENSITY}

In this section, we briefly outline the NMSSM model setup relevant to our scenario, which provides light singletlike Higgs bosons and a light singlinolike neutralino as a DM candidate with right relic density [Eq. (1.1)]. The NMSSM contains an additional gauge singlet superfield (S) along with two Higgs doublet superfields $\left(H_{u}\right.$ and $\left.H_{d}\right)$. The corresponding $Z_{3}$-invariant superpotential is given by $[25-28,57]$,

$$
W_{\mathrm{NMSSM}}=W_{\mathrm{MSSM}}+\lambda S H_{u} H_{d}+\frac{1}{3} \kappa S^{3},
$$

where $\lambda$ and $\kappa$ are the dimensionless couplings, and $W_{\text {MSSM }}$ represents the part of the superpotential in MSSM counting Higgs doublets but without the $\mu$-term. In addition, two soft terms, $\lambda A_{\lambda} S H_{u} H_{d}$ and $\frac{1}{3} \kappa A_{\kappa} S^{3}$ are also included. The Yukawa-like term with coupling $\lambda$ generates the Higgsino mass term, $\mu_{\text {eff }}=\lambda v_{s}$, where $v_{s}$ is the vacuum expectation value (VEV) acquired by the singlet superfield. The dynamic generation of the $\mu$-term, the key aspect for the motivation of NMSSM, prevents it from acquiring a large value [29]. The Higgsino mass term is expected to be at the level of the electroweak scale to obtain appropriate electroweak symmetry breaking [27]. On the other hand, phenomenologically, $\mu_{\text {eff }}$ is restricted to be $\mu_{\text {eff }} \gtrsim 100 \mathrm{GeV}$, due to the chargino mass limit predicted by LEP experiment [58].

As mentioned before, the enlarged Higgs sector of NMSSM consists of seven physical Higgs bosons, $3 C P$ even states $\left(H_{1}, H_{2}, H_{3}\right.$, assuming $\left.m_{H_{1}}<m_{H_{2}}<m_{H_{3}}\right)$ and $2 C P$ odd states $\left(A_{1}, A_{2}\right.$, assuming $\left.m_{A_{1}}<m_{A_{2}}\right)$ and 2 charged Higgs $\left(H^{ \pm}\right)$boson states. Masses and couplings of these Higgs bosons are determined by model parameters. The Higgs sector is briefly revisited here to identify respective ranges of corresponding parameters to our interest. The $3 C P$ even Higgs states are described by $3 \times 3$ mass matrices in the basis $\psi_{R} \equiv\left(H_{u R}, H_{d R}, S_{R}\right)$, the real parts of Higgs fields. The elements of the mass matrix are given by [28],
$M_{S, 11}^{2}=M_{Z}^{2} \sin ^{2} \beta+\mu_{\text {eff }} \cot \beta\left(A_{\lambda}+\kappa v_{s}\right)$,

$M_{S, 22}^{2}=M_{Z}^{2} \cos ^{2} \beta+\mu_{\text {eff }} \tan \beta\left(A_{\lambda}+\kappa v_{s}\right)$,

$M_{S, 33}^{2}=\frac{\lambda^{2} v^{2} A_{\lambda} \sin 2 \beta}{2 \mu_{\mathrm{eff}}}+\kappa v_{s}\left(A_{\kappa}+4 \kappa v_{s}\right)$,

$M_{S, 12}^{2}=\left(\lambda^{2} v^{2}-\frac{M_{Z}^{2}}{2}\right) \sin 2 \beta-\mu_{\mathrm{eff}}\left(A_{\lambda}+\kappa v_{s}\right)$,

$M_{S, 13}^{2}=\lambda v\left(2 \mu_{\mathrm{eff}} \sin \beta-\left(A_{\lambda}+2 \kappa v_{s}\right) \cos \beta\right)$,

$M_{S, 23}^{2}=\lambda v\left(2 \mu_{\mathrm{eff}} \cos \beta-\left(A_{\lambda}+2 \kappa v_{s}\right) \sin \beta\right)$.

Here $\tan \beta$ is the ratio of VEVs of neutral components of two Higgs doublet. The masses of $3 C P$-even Higgs boson states can be obtained by diagonalizing the mass matrix by an orthogonal matrix $\left(S_{i j} ; i, j=1-3\right)$, and hence physical states $\left(H_{i}\right)$ become the admixture of weak Higgs boson states as,

$$
H_{i}=\sum_{j=1}^{3} S_{i j} \psi_{j R} .
$$

Notably, the lightest $C P$-even Higgs boson mass is found to be bounded by $[59,60], m_{H_{1}}^{2} \leq M_{Z}^{2} \cos ^{2} 2 \beta+\lambda^{2} v_{s}^{2} \sin ^{2} 2 \beta$ at the tree level. Notice that the extra contribution lifts the tree level Higgs boson mass substantially, and hence may not require a huge contribution from higher-order correction [61]. As a consequence, a wide region of parameter space which is less constrained can easily accommodate one of the $\mathrm{CP}$-even Higgs boson (primarily either $H_{1}$ or $\mathrm{H}_{2}$ ) states as the SM-like Higgs boson with a mass $\sim 125 \mathrm{GeV}$. This feature makes the NMSSM very attractive after the discovery of the SM Higgs boson at the LHC [33,61-64].

In the $C P$-odd sector, eliminating the Goldstone modes, the elements of $2 \times 2$ mass matrix for $C P$-odd Higgs boson states in $\psi_{I} \equiv\left(A, S_{I}\right)$ basis are given as,

$$
\begin{aligned}
& M_{P, 11}^{2}=\frac{2 \mu_{\mathrm{eff}}}{\sin 2 \beta}\left(A_{\lambda}+\kappa v_{s}\right), \\
& M_{P, 22}^{2}=\lambda^{2} v^{2} \frac{\sin 2 \beta}{2 \mu_{\mathrm{eff}}}\left(A_{\lambda}+4 \kappa v_{s}\right)-3 A_{\kappa} \kappa v_{s}, \\
& M_{P, 12}^{2}=\lambda v\left(A_{\lambda}-2 \kappa v_{s}\right) .
\end{aligned}
$$

Similarly, diagonalizing this mass matrix by an orthogonal $\left(P_{i j}, i, j=1,2\right)$ matrix, the masses of the two physical $C P$-odd states $\left(A_{1}, A_{2}\right)$ can be obtained, and hence the corresponding composition of physical states are given as,

$$
A_{i}=\sum_{j=1}^{2} P_{i j} \psi_{j I} .
$$

Interestingly, unlike the MSSM, in NMSSM, the physical Higgs boson states contain a fraction of the singlet 
component $\left(S_{I}\right)$ which does not couple with fermions and gauge bosons. Of course, the content of singlet component in physical states is very much parameter space sensitive.

The Higgs sector and the corresponding masses and composition of physical states are described by six parameters:

$$
\lambda, \quad \kappa, \quad A_{\lambda}, \quad A_{\kappa}, \quad \tan \beta, \quad \mu_{\text {eff }} .
$$

Dependence on squark masses and other trilinear terms (A-terms) occurs via radiative corrections[65].

The fermionic superpartner $(\tilde{S})$ of the singlet field, mixes with Higgsinos extending the neutralino mass matrix to $5 \times 5$, in the basis $\left(-i \tilde{B},-i \tilde{W}_{3}, \tilde{H}_{u}^{0}, \tilde{H}_{d}^{0}, \tilde{S}\right)$ and it is presented as,

$$
M_{N}=\left(\begin{array}{ccccc}
M_{1} & 0 & \frac{-g_{1} v s_{\beta}}{\sqrt{2}} & \frac{g_{1} v c_{\beta}}{\sqrt{2}} & 0 \\
0 & M_{2} & \frac{g_{2} v s_{\beta}}{\sqrt{2}} & \frac{-g_{2} v c_{\beta}}{\sqrt{2}} & 0 \\
\frac{-g_{1} v s_{\beta}}{\sqrt{2}} & \frac{g_{2} v s_{\beta}}{\sqrt{2}} & 0 & -\mu_{\mathrm{eff}} & -\lambda v c_{\beta} \\
\frac{g_{1} v c_{\beta}}{\sqrt{2}} & \frac{-g_{2} v c_{\beta}}{\sqrt{2}} & -\mu_{\mathrm{eff}} & 0 & -\lambda v s_{\beta} \\
0 & 0 & -\lambda v s_{\beta} & -\lambda v c_{\beta} & 2 \kappa v_{s}
\end{array}\right)
$$

with $s_{\beta} \equiv \sin \beta, c_{\beta} \equiv \cos \beta, M_{1}$ and $M_{2}$ are the masses of $\tilde{B}$ and $\tilde{W}_{3}$ gauginos respectively, $v_{u}$ and $v_{d}$ are the VEVs for neutral components of $H_{u}$ and $H_{d}$ fields and are constrained to be $v_{u}^{2}+v_{d}^{2}=v^{2} ; g_{1}$ and $g_{2}$ are weak couplings. The masses of 5 neutralino states, $m_{\tilde{\chi}_{i}^{0}}(i=1, \ldots, 5)$ can be obtained by diagonalizing the mass matrix $M_{N}$ by an orthogonal matrix $N_{5 \times 5}$ as,

$$
M_{\tilde{\chi}^{0}}^{D}=N M_{N} N^{\dagger} .
$$

The analytical expressions of $m_{\tilde{\chi}_{i}^{0}}$ and the corresponding physical states exist in the literature for the MSSM [66,67], and as well as for the NMSSM [68,69]. The masses and couplings of neutralinos are very sensitive to NMSSM specific parameters, in particular $\lambda, \kappa$ and $v_{s}$ or $\mu_{\text {eff }}$, along with $M_{1}$ and $M_{2}$. Moreover, these parameters (except $M_{1}$ and $M_{2}$ ) are also strongly connected with the Higgs sector [Eq. (2.2)-(2.4)], and play important roles, along with $A_{\lambda}$ and $A_{\kappa}$ in determining the masses and mixings of Higgs bosons.

As stated earlier, the goal of this study is to provide a low mass DM solution within the framework of the NMSSM. With this motivation, we try to identify the corresponding regions of relevant model parameters compatible with all existing experimental constraints.

In our proposed solution, DM annihilation takes place via s-channel mediated by light Higgs scalars giving a pair of fermions in the final state [70-72],

$$
\chi \chi \rightarrow H_{1} / A_{1} \rightarrow f \bar{f} .
$$

The DM annihilation rate is primarily sensitive to the interaction between neutralino pair and Higgs boson, and their relative mass difference. The Higgs-neutralino-neutralino couplings are given as[28,38],

$$
\begin{aligned}
g_{\tilde{\chi}_{1}^{0} \tilde{\chi}_{1}^{0} H_{i}}= & \sqrt{2} \lambda N_{15}\left(S_{i 1} N_{14}+S_{i 2} N_{13}\right) \\
& +\sqrt{2} S_{i 3}\left(\lambda N_{13} N_{14}-\kappa N_{15}^{2}\right) \\
& -\frac{g}{2}\left(N_{12}-\tan \theta_{w} N_{11}\right)\left(S_{i 1} N_{13}-S_{i 2} N_{14}\right),
\end{aligned}
$$

$$
\begin{aligned}
g_{\tilde{\chi}_{1}^{0} \tilde{\chi}_{1}^{0} A_{i}}= & \sqrt{2} \lambda N_{15}\left(P_{i 1} N_{14}+P_{i 2} N_{13}\right) \\
& +\sqrt{2} P_{i 2}\left(\lambda N_{13} N_{14}-\kappa N_{15}^{2}\right) \\
& -\frac{g}{2}\left(N_{12}-\tan \theta_{w} N_{11}\right)\left(P_{i 1} N_{13}-P_{i 2} N_{14}\right) .
\end{aligned}
$$

Here $N_{15}$ presents the singlino composition of the lightest neutralino, whereas $S_{i 3}$ and $P_{i 2}$ stand for the singlet components of $H_{i}$ and $A_{i}$ respectively. Parameters $\lambda$ and $\kappa$, which are connected with the singlino mass and its composition, are found to be very sensitive to the annihilation cross section due to the above couplings [Eq. (2.10) and (2.11)]. The analytical expressions for the cross section of annihilation processes are presented in Appendix A. As indicated, the right relic density corresponding to the lower range $(\lesssim 20 \mathrm{GeV})$ of DM masses can be achieved by requiring neutralino and Higgs boson states singlino and singlet dominated respectively (i.e., $N_{15}, P_{12}, S_{13} \sim 1$ ), for which

$$
\begin{gathered}
g_{\tilde{\chi}_{1}^{0} \tilde{\tilde{1}}_{1}^{0} H_{1}} \sim \sqrt{2} S_{13}\left(\lambda N_{13} N_{14}-\kappa N_{15}^{2}\right) \sim-\sqrt{2} S_{13} \kappa N_{15}^{2}, \\
g_{\tilde{\chi}_{1}^{0} \tilde{\chi}_{1}^{0} A_{1}} \sim-\sqrt{2} P_{12} \kappa N_{15}^{2} .
\end{gathered}
$$

The DM-nucleon scattering cross sections, both $\sigma_{\mathrm{SI}}$ and $\sigma_{\mathrm{SD}}$ mediated by Higgs scalars and gauge bosons respectively, are given in Appendix B. From direct searches, allowed spinindependent cross section corresponding to DM masses of our interest, varies from $\sim 10^{-44} \mathrm{~cm}^{2}-10^{-46} \mathrm{~cm}^{2}$, which is achievable through the adjustments of coupling $g_{\tilde{\chi}_{1}^{0} \tilde{\chi}_{1}^{0} H_{1}}$ or $g_{\tilde{\chi}_{1}^{0}} \tilde{\chi}_{1}^{0} A_{1}$. Again, we observed that a singlinolike lightest neutralino and singlet dominant light Higgs bosons are most favored. It suggests that the light singlinolike DM candidate requires singlet dominated light Higgs boson states in order to have right relic density and DM-nucleon scattering cross section [3,4,7-9]. Therefore, the preferred parameter space favoring our scenario should provide, (a) a light singlinolike LSP, (b) light singletlike Higgs boson states. 
A closer look at the neutralino mass matrix reveals few features of neutralino masses and mixings [57]. For instance, the absence of mixing terms between singlino and gaugino fields implies no interaction between singlinolike neutralino and gauginolike or gauge boson states. Notice that the mixing between singlet and doublet Higgs fields is decided by $\lambda v \cos \beta$ or $\lambda v \sin \beta$ [Eq. (2.7)]. Among the five neutralino states, two of them remain to be gauginolike if, $\left|M_{1,2}-\mu_{\text {eff }}\right| \geq M_{Z}$, the mass of $Z$-boson. For a decoupling scenario, $2|\kappa| v_{s} \ll \mu_{\mathrm{eff}}, M_{1,2}$, the mass of singlinolike neutralino turns out to be $\sim 2|\kappa| v_{s}$, and dominantly a singlinolike. On the other hand, since $\mu_{\text {eff }}$ or $\lambda v_{s} \sim \mathcal{O}(100) \mathrm{GeV}$, hence for smaller values of $\lambda \lesssim 0.1$, the typical value of $v_{s}$ is expected to be large $\sim \mathcal{O}(1) \mathrm{TeV}$. Therefore, for a very light singlinolike LSP, $|\kappa|$ should lie within the range of $\sim 10^{-3}$. For higher values of $\lambda \sim 0.1$, it is possible to accommodate comparatively lower values of $v_{s}$, with little larger values of $|\kappa| .^{1}$ In fact, the mass of singlinolike LSP, $m_{\tilde{\chi}_{1}^{0}} \sim 2\left(\frac{\kappa}{\lambda}\right) \mu_{\text {eff }}$ becomes small for $\frac{\kappa}{\lambda} \sim 10^{-2}$. On the contrary, for $2|\kappa| v_{s} \gg M_{1,2}, \mu_{\text {eff }}$, singlinolike state becomes very heavy, and decouples from other neutralino states which consist of only Higgsino and gaugino components like MSSM scenario. The other NMSSM parameters $A_{\kappa}$ and $A_{\lambda}$, which are not related with neutralino masses and mixings at the tree level, are expected to be restricted due to the requirements of light singletlike Higgs bosons. Following Eq. (2.4), the lighter $C P$ odd state $\left(A_{1}\right)$ is found to be singletlike for decoupling type of scenario such as [73],

$$
M_{P, 11}^{2}>M_{P, 12}^{2}, M_{P, 22}^{2},
$$

which also leads heavier state $\left(A_{2}\right)$ MSSM like. ${ }^{2}$ Hence, $A_{\lambda}$ is preferred to be very large $(\sim 2-3 \mathrm{TeV})$ [see Eq. (2.4)], since $2|\kappa| v_{s}$ is required to be very small, to have a light LSP. With a good approximation, one can obtain mass of $A_{1}$ as $m_{A_{1}}^{2} \simeq-3 A_{\kappa} \kappa v_{s}$ [57]. Moreover, as required above, $|\kappa| v_{s}$ cannot be large, so a moderate range $(\mathcal{O}(10) \mathrm{GeV})$ of $A_{\kappa}$ is required to obtain a light $A_{1}$ state. For the $C P$ even Higgs sector, the spectrum of relevant parameters corresponding to our interest can be understood following a sum rule obtained using the tree level masses of $H_{1}$ and $H_{2}$. This sum rule reads as [57],

$$
m_{H_{1}}^{2}+m_{H_{2}}^{2} \equiv M_{Z}^{2}+\frac{1}{2} \kappa v_{s}\left(4 \kappa v_{s}+\sqrt{2} A_{\kappa}\right) .
$$

Naturally, if any of the Higgs boson states (here it is $\mathrm{H}_{2}$ ) becomes massive and close to the mass of the SM-like

\footnotetext{
${ }^{1}$ Requirements of both $\kappa$ and $\lambda$ remain to be perturbative up to GUT scale impose the constraint $\lambda^{2}+\kappa^{2} \lesssim 0.5$ [57].

${ }^{2}$ Similar scenario can also occur if off-diagonal entry $M_{P, 12}^{2} \sim 0$. But it is not a viable option to our interest as $\kappa v_{s}$ is required to be very small.
}

Higgs boson, then for a moderate value of $A_{\kappa}, H_{1}$ state becomes very light, even may be less than the half of the mass of $\mathrm{H}_{2}$ state. Since $\mathrm{H}_{2}$ state is SM-like, hence mixing between singlet and doublet components $\left(H_{u}, H_{d}\right)$ should be very small, yielding an $H_{1}$ state that is mostly singlet dominated. Furthermore, since the annihilation process occurs via s-channel Higgs exchange, the cross section enhanced significantly, for $m_{A_{1} / H_{1}} \sim 2 \times m_{\tilde{\chi}_{1}^{0}}$, which we also require for our proposed collider searches. The third $\mathrm{CP}$ even physical Higgs state $H_{3}$, seems to be very massive and decoupled for large values of $A_{\lambda}$. Finally, with all these above arguments corresponding to our proposed scenario, we conclude:

(i) light singlinolike LSP requires very small $|\kappa| v_{s}$, with $\kappa / \lambda \sim 10^{-2}$,

(ii) requirement of light Higgs boson states to be singletlike, leads $A_{\lambda}$ to be very large(few $\mathrm{TeV}$ ), but $A_{\kappa}$ not necessarily to be very large, but with a relative sign opposite to $\kappa$.

\section{PARAMETER SCAN}

Probable regions of parameters interesting to us are identified performing a naive numerical scan using NMSSMTools [74,75], interfaced with micrOMEGAs [37,76-78] for calculation of DM observables. For the random scan, the numerical ranges of six sensitive parameters [Eq. (2.6)] are set as:

$$
\begin{aligned}
& 0.1 \leq \lambda \leq 0.65, \quad-0.01 \leq \kappa \leq 0.01, \\
& 1.5 \leq \tan \beta \leq 20, \quad 100 \mathrm{GeV} \leq \mu_{\text {eff }} \leq 1000 \mathrm{GeV},
\end{aligned}
$$

$500 \mathrm{GeV} \leq A_{\lambda} \leq 3500 \mathrm{GeV}$,

$-100 \mathrm{GeV} \leq A_{\kappa} \leq 100 \mathrm{GeV}$.

We first performed a scan for a very wide range of these set of parameters, and then focus only on the above narrow range which is relevant to the signal phenomenology to be studied in this paper. The A-term for third generation $\left(A_{t}\right)$ plays an important role in predicting the mass of the SMlike Higgs boson $[28,65]$ and is varied for a wider range,

$$
-8 \mathrm{TeV}<A_{t}<+8 \mathrm{TeV},
$$

while setting other 3rd generation trilinear parameters as,

$$
A_{b}=2 \mathrm{TeV} \quad \text { and } \quad A_{E_{3}}=1.5 \mathrm{TeV} \text {. }
$$

In order to reduce the number of parameters to vary, all soft masses for left and right handed squarks for the first two generations are assumed as,

$$
\begin{gathered}
M_{Q_{1,2}}=M_{U_{1,2}}=M_{D_{1,2,3}}=1 \mathrm{TeV} . \\
1 \mathrm{TeV} \leq M_{Q_{3}}, \quad M_{U_{3}} \leq 4 \mathrm{TeV}
\end{gathered}
$$


The gaugino masses $M_{1}, M_{2}$ and $M_{3}$, which are important for chargino and neutralino sectors are set to be within the range,

$100 \mathrm{GeV} \leq M_{1} \leq 1 \mathrm{TeV}, \quad 100 \mathrm{GeV} \leq M_{2} \leq 1 \mathrm{TeV}$,

$100 \mathrm{GeV} \leq M_{3} \leq 2 \mathrm{TeV}$.

Slepton masses of first two generations are fixed to,

$$
M_{L_{1,2}}=300 \mathrm{GeV}, \quad M_{E_{1,2}}=300 \mathrm{GeV} .
$$

While performing the numerical scan, various constraints, theoretical and as well as experimental, included in NMSSMTools5.5.0 [74,75] are examined, and accordingly, mass points are rejected or accepted. Precision measurements of the SM-like Higgs boson are used to constrain the model along with the mass requirement of $125 \pm 3 \mathrm{GeV}$. In addition, limits on supersymmetric particles obtained at LEP, and Tevatron experiments, and as well as at the LHC are also imposed. Various measurements in flavor physics are also used to check the consistency of mass points. Of course, since the lightest neutralino is assumed to be a DM candidate, it is also ensured that the selected mass points are consistent with PLANCK [3] constraint and Direct searches [4-9]. It is to be noted that the numerical (random) scan performed in this study is a representative one. The main goal of this scan is to identify potential region of parameters interesting to us, and then use few points of the allowed space as benchmark parameters to present the results. Certainly, one needs to perform more rigorous scan in order to find the complete numerical range of parameters through a more sophisticated method, e.g., those described in Ref. [79-81].

In the following, we present the allowed range of sensitive parameters, which are mentioned in the previous section. We focus the region of parameters which provide the mass of the lightest singlinolike neutralino up to $25 \mathrm{GeV}$ and lightest Higgs bosons almost twice the singlino mass. In Fig. 1, the spin-independent(SI) DM-nucleon cross sections are presented (dotted) for a range of neutralino masses up to $25 \mathrm{GeV}$ and it is also subject to XENON1T and PICO constraints [4,9]. It clearly demonstrates that the lightest neutralino, even with reasonably low mass, can emerge as a viable DM candidate in the NMSSM. In Fig. 2, we show the dependence of lightest neutralino mass corresponding to the interesting range shown in Fig. 1, on $\frac{\kappa}{\lambda}$ and $\mu_{\text {eff }}$. As anticipated, preferred values are $|\kappa| \sim 10^{-3}$ and $\lambda \sim 10^{-1}$, whereas $\mu_{\text {eff }} \lesssim 1 \mathrm{TeV}$, are not expected to be very large.

The trilinear parameters $A_{\kappa}$ and $A_{\lambda}$, play a crucial role along with $\kappa$ and $\lambda$, in determining the masses of Higgs bosons [57], in particular, $m_{H_{1}}$ and $m_{A_{1}}$. In Fig. 3, the available region in the $A_{\kappa}-\kappa$ and $A_{\lambda}-\kappa$ plane, relevant to our scenario, are presented along with $\mu_{\text {eff }}$. As argued above, for a very small value of $\kappa$, the large values of $A_{\lambda} \sim \mathcal{O}(1000) \mathrm{GeV}$ and $A_{\kappa} \sim \mathcal{O}(10) \mathrm{GeV}$ are required

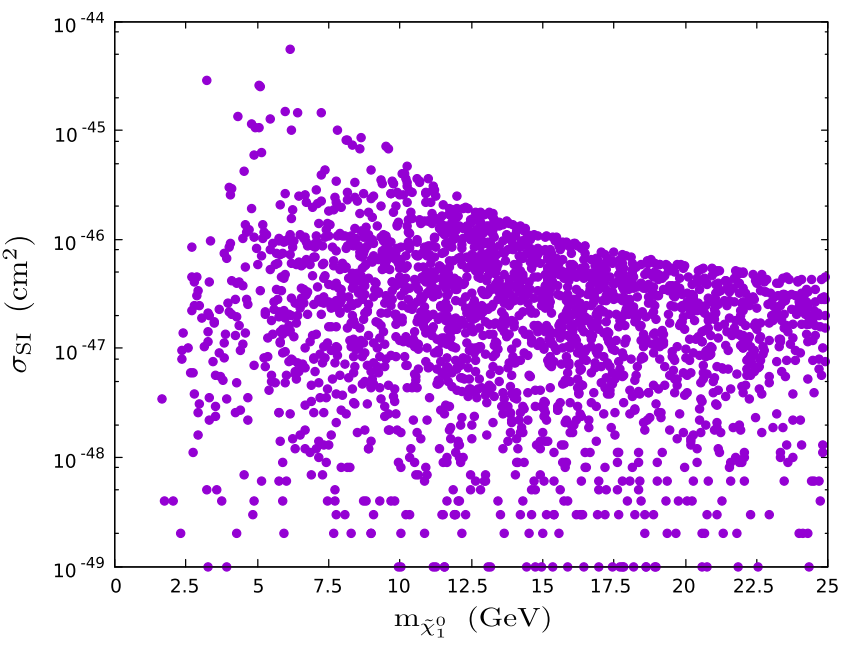

FIG. 1. Allowed regions (dotted) of SI DM-nucleon scattering cross section with the variation of $\tilde{\chi}_{1}^{0}$ masses.

corresponding to our considered scenario. The value of $|\kappa| \sim 0$ is not permissible and the symmetric nature of distribution arises because of the dependence of the value of $\kappa$. We have checked that corresponding to this parameter space (Figs. 1-3), the singlet composition in lighter Higgs boson states, and singlino content in lightest neutralino, both are at the level of $95 \%$ or more.

Branching fractions for $H_{\mathrm{SM}} \rightarrow H_{1} H_{1} / A_{1} A_{1}$ and subsequent decays, $H_{1} / A_{1} \rightarrow \tilde{\chi}_{1}^{0} \tilde{\chi}_{1}^{0}$ or $f \bar{f}$ decide the signal rate. We observe that for a favored range of parameters, such as $\lambda, \kappa, A_{\lambda}$ and $A_{\kappa}$, as discussed above, the $\operatorname{BR}\left(H_{\mathrm{SM}} \rightarrow\right.$ $\left.H_{1} H_{1} / A_{1} A_{1}\right) \sim 10 \%$ or less, which is much below the upper limit of $\mathrm{BR}\left(H_{\mathrm{SM}} \rightarrow \mathrm{BSM}\right)$, constrained by Higgs data, and given by [82],

$$
\mathrm{BR}_{\mathrm{BSM}}<0.26 \text { at } 95 \% \text { C.L. }
$$

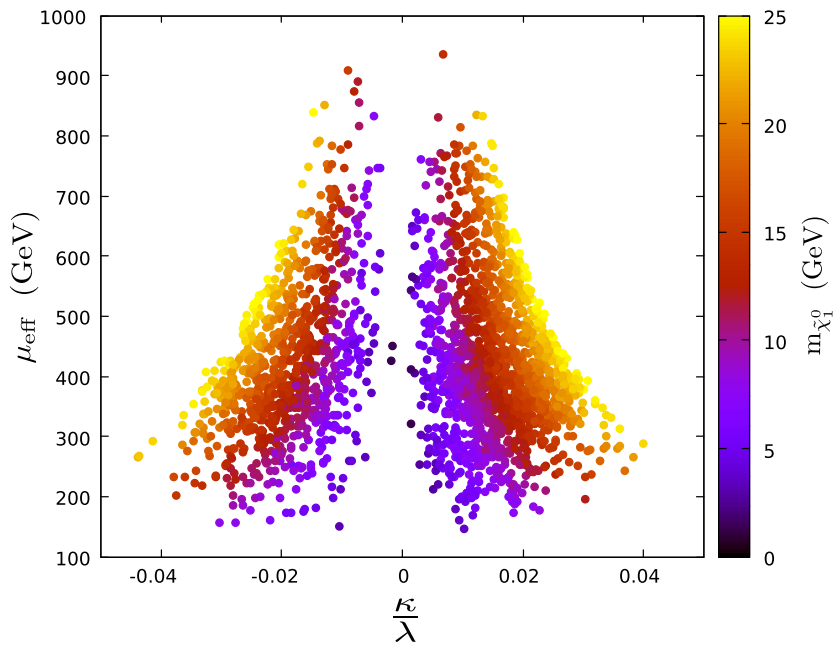

FIG. 2. Allowed regions (dotted) in the $\mu_{\text {eff }}$ and $\frac{\kappa}{\lambda}$ plane with the $m_{\tilde{\chi}_{1}^{0}}$. 

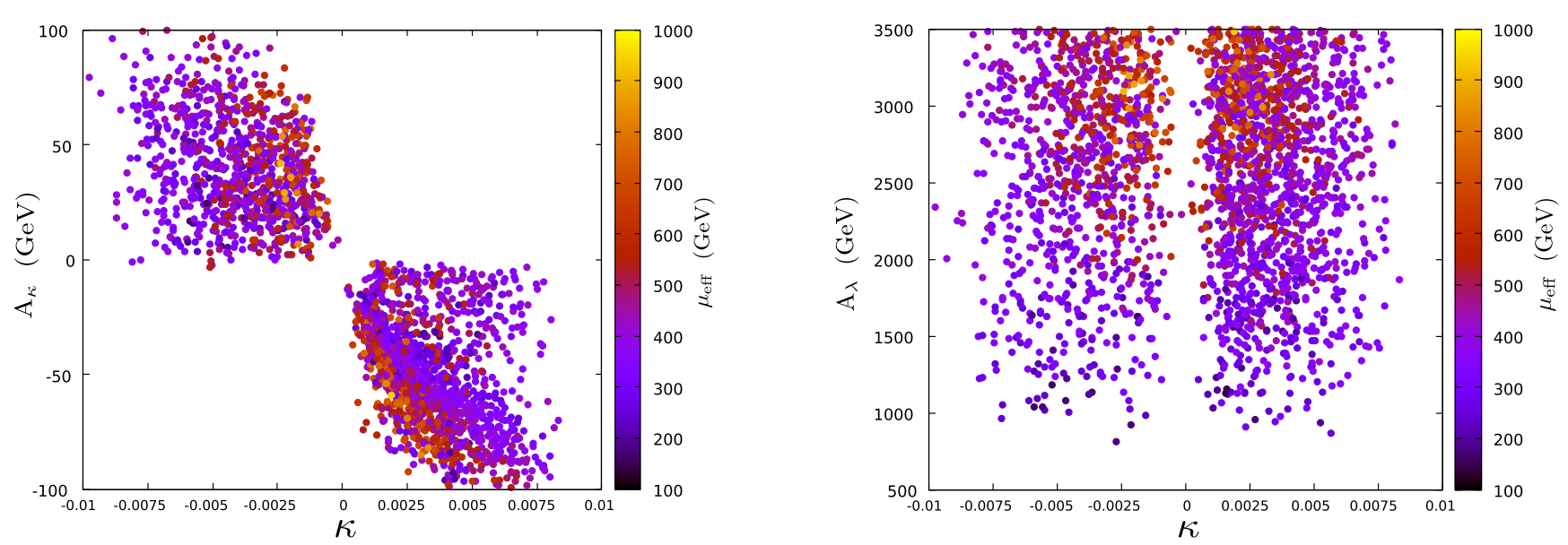

FIG. 3. Allowed regions (dotted) in the $\kappa-A_{\kappa}$ (left) and $\kappa-A_{\lambda}$ (right) plane with $\mu_{\text {eff }}$.

Branching ratio of light Higgs bosons decay to LSP is also very sensitive to $\lambda$ and $\kappa$, as evident from Eq. (2.12) and (2.13). A substantial amount of singlet composition in light Higgs boson state and singlino content in LSP favor this decay channel. However, even a little presence of doublet components in light Higgs bosons enhance the decay rate in the fermionic channel $(f \bar{f})$. Corresponding to our interesting region of parameters, the $\operatorname{BR}\left(H_{1} / A_{1} \rightarrow \tilde{\chi}_{1}^{0} \tilde{\chi}_{1}^{0}\right)$ appears to be quite reasonable, and sometimes it turns out to be around $\sim 70-80 \%$.

\section{SIGNAL AND BACKGROUND}

In this section, we present the discovery potential of singlinolike DM signal at the LHC with the CM energy $\sqrt{s}=14 \mathrm{TeV}$ for few luminosity options. We consider the production of light singletlike Higgs bosons via the nonstandard decay channel of the SM Higgs, $H_{\mathrm{SM}} \rightarrow H_{1} H_{1} / A_{1} A_{1}$, where the mass of $H_{1}$ or $A_{1}$ is less than the half of the mass of the SM Higgs boson. Subsequently, the lighter Higgs boson states are assumed to decay to lightest neutralino pair $\left(H_{1} / A_{1} \rightarrow \tilde{\chi}_{1}^{0} \tilde{\chi}_{1}^{0}\right)$ with a reasonable $\mathrm{BR}$ depending on the model parameter space, whereas the other competitive decay modes are to heavy fermions, like $b \bar{b}$ when kinematically accessible, otherwise $\tau \tau$. To ensure harder final state particles, we focus on exclusive $H_{\mathrm{SM}}+1$ jet process. As we know, the most dominant process of Higgs production proceeds via heavy top quark loop leading to, $g g \rightarrow H_{\mathrm{SM}}[53,54]$. An additional jet originates in next-to-leading order(NLO) perturbative QCD with a significant increase of cross section, either from initial gluons or the heavy quarks inside the loop, leading to $g g \rightarrow H_{\mathrm{SM}}+g$. Hence, the signal process to our interest appears to be,

$$
\begin{aligned}
g g & \rightarrow H_{\mathrm{SM}}+\text { jet } \rightarrow H_{1} H_{1} / A_{1} A_{1}+\text { jet } \\
& \rightarrow b \bar{b} \quad \text { or } \quad \tau \tau+\tilde{\chi}_{1}^{0} \tilde{\chi}_{1}^{0}+\text { jet }
\end{aligned}
$$

Thus, we focus on signal final state comprising missing energy, which is a characteristic of DM signature, along with a reconstructed Higgs boson mass accompanied with at least one untagged jet.

The separation between decay products from lighter Higgs boson is given by [55],

$$
\Delta R(f, \bar{f}) \simeq \frac{m_{A_{1} / H_{1}}}{z(z-1) p_{T}},
$$

implying they are collimated for larger $p_{T}$ and/or lower mass of parent particle, where $\mathrm{z}$ is the fraction of momentum of Higgs boson carried by one of the decay product. In Fig. 4, we demonstrate the transverse momentum of lighter Higgs boson originating from SM Higgs decay (left) and the separation [Eq. (4.2)] between their decay products (right) for three sets of Higgs boson masses.

Clearly, the lighter states are more boosted and their decay products are more collimated than those from higher states. These characteristic kinematic features are exploited in simulation to isolate signal. Armed with this observation, simulation is performed for the signal setting three ranges of the mass of $H_{1}$ or $A_{1}$, as: (i) lower mass region: $m_{H_{1} / A_{1}} \leq$ $10 \mathrm{GeV}$, (ii) moderate mass region: $10 \mathrm{GeV} \leq m_{H_{1} / A_{1}} \leq$ $30 \mathrm{GeV}$, and (iii) higher mass region: $30 \mathrm{GeV} \leq m_{H_{1} / A_{1}} \leq$ $60 \mathrm{GeV}$.

Notably, as stated above, for "low" and "moderate" mass regions, the decay products, either $\tau \tau$ or $b \bar{b}$ pair appears to be very collimated, and emerge as a single "Higgs jet" $(\mathrm{HJ})$ with constituents either two $b$-like $\left(J_{b \bar{b}}\right)$ or $\tau\left(J_{\tau \tau}\right)$-like subjets depending on the decay modes. Hence, instead of tagging individual $\tau$-jet or $b$-jet, which is challenging in this present scenario, Higgs jet is tagged to classify signal from the background. On the contrary, tagging HJ is not very effective for "high mass region," since decay products emerge with a wider separation. In this case, we observed that even losing signal events due to tagging of $\mathrm{HJ}$, still it is very useful to reduce the SM backgrounds substantially. 

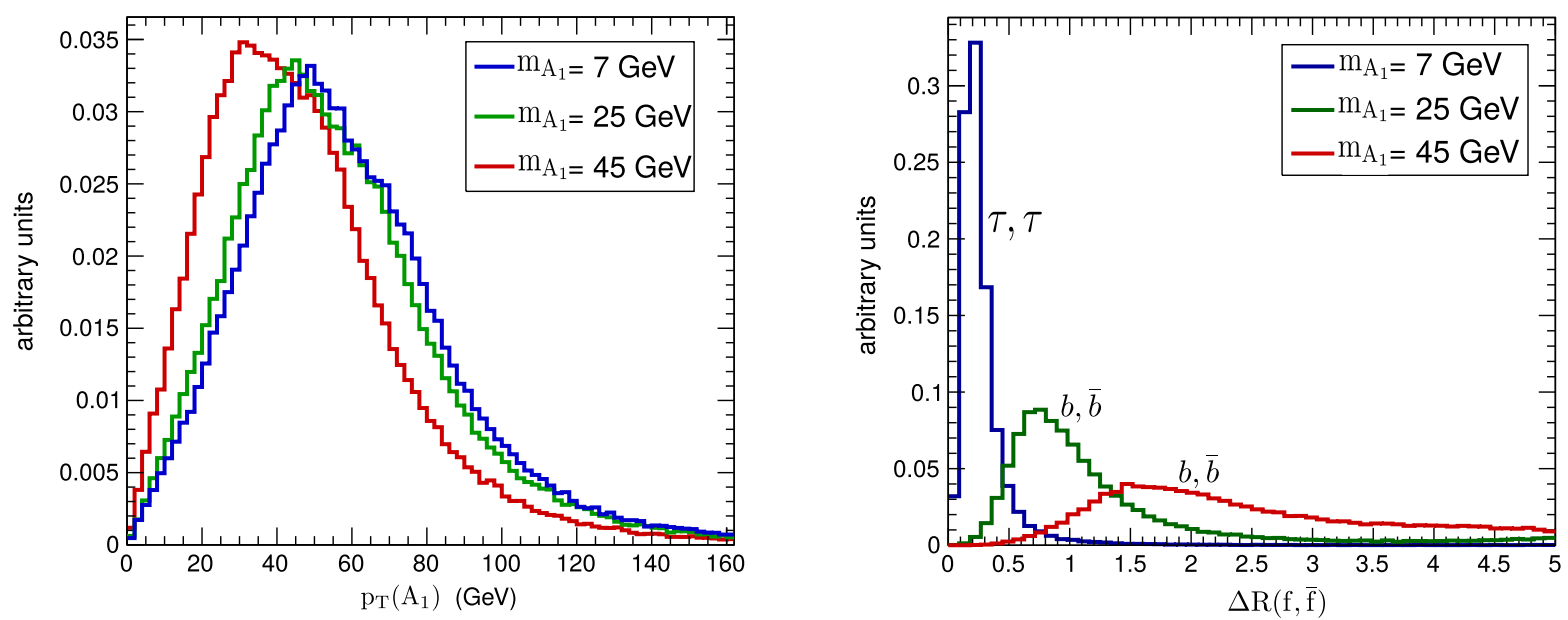

FIG. 4. Transverse momentum of light Higgs boson (left) and $\Delta R$ [Eq (4.2)] (right) between two fermions originating from the decay of light Higgs bosons.

Hence, in summary, simulation is performed for three categories:

$$
\begin{array}{r}
J_{\tau \tau}+\not E_{T}+\geq 1 j \text { for } m_{H_{1} / A_{1}} \leq 10 \mathrm{GeV}, \\
J_{b \bar{b}}+\not E_{T}^{\dagger}+\geq 1 j \text { for } 10 \mathrm{GeV} \leq m_{H_{1} / A_{1}} \leq 30 \mathrm{GeV}, \\
J_{b \bar{b}}+\not_{T}^{\dagger}+\geq 1 j \text { for } 30 \mathrm{GeV} \leq m_{H_{1} / A_{1}} \leq 60 \mathrm{GeV} .
\end{array}
$$

We discuss signal selection strategy for the case of lower mass range, Eq. (4.3), in a later subsection separately.

The dominant sources of SM backgrounds corresponding to the signal processes [Eq. (4.4) and (4.5)] are due to the processes:

$$
p p \rightarrow t \bar{t}, \quad W b \bar{b}+\text { jets, } \quad Z b \bar{b}+\text { jets. }
$$

Neutrinos originating from $W$ or $Z$ decay contribute to missing transverse energy $\left(E_{T}\right)$. We also checked the level of background contribution from $W Z j, Z Z j, H_{\mathrm{SM}} W j$ and $H_{\mathrm{SM}} Z j\left(H_{\mathrm{SM}} \sim H_{2}\right)$ and found to be very small due to comparatively very low cross sections and respective branching ratios.

For the sake of illustration, six benchmark points (BP), as shown in Table I, compatible with various experimental data, are chosen to simulate the signal process. These BPs are selected such that $2 m_{\tilde{\chi}_{1}^{0}} \sim m_{H_{1} / A_{1}}$ and covering mass ranges as required in Eq. (4.3)-(4.5). Notice that for all such cases, $H_{2}$ turns out to be the SM-like Higgs boson and decays to a pair of non-SM-like Higgs bosons states $H_{2} \rightarrow H_{1} H_{1} / A_{1} A_{1}$, with a BR ranging from $\sim 0.01 \%$ to $10 \%$, which is within the constraint given by Eq. (3.8). As mentioned before, light Higgs bosons, mainly decay to either in $b \bar{b}$ or $\tilde{\chi}_{1}^{0} \tilde{\chi}_{1}^{0}$ channel, which we require for our signal process.
PYTHIA8 is used to simulate $t \bar{t}$ events, while other processes are generated using MadGraph5-aMC@NLO-2.6.4 [83] and PYTHIA8 $[84,85]$ for subsequent showering and hadronization. The signal events are generated using PYTHIA8 inputting masses and branching ratios of SUSY particles and Higgs bosons through SLHA file [86] which is generated using NMSSMTools. To take into account detector effects, generated events for both signal and backgrounds are passed through DELPHES-3.4.2 [87] using the CMS detector card. The DELPHES objects, namely, eflows are used for analysis.

In the simulation, events are selected adopting the following strategy.

(i) Lepton veto: Events consisting leptons are vetoed out. Leptons are selected with $p_{T}^{\ell}>10 \mathrm{GeV}$ and $|\eta|<2.5$. It reduces the background events significantly without losing any signal.

(ii) HJ selection: The e-flow objects (e-flow tracks, e-flow photons and e-flow neutral hadrons) of DELPHES are given as input to FastJet3.3.2 [88] to construct fat jets. The Cambridge-Aachen [89] algorithm is used setting the jet size parameter $R=1$ and 1.6 for moderate and high mass regions [Eq. (4.4) and (4.5)] of lighter Higgs bosons respectively. The Fatjets are selected with $p_{T}^{J}>40 \mathrm{GeV}$ and $|\eta|<4$ 4.0. Mass-drop tagger (MDT) [55,90] with $\mu=0.667$ and $y_{\text {cut }}>0.01$ is used to tag Fat-jets with two subjets. The subjets of "tagged fat jet" are further matched with the $b$-quarks of the events which are selected with a minimum $p_{T}$ cut of $0.5 \mathrm{GeV}$ and $|\eta|<2.5$ with a matching cone $\Delta R<0.3$, where $\Delta R=\sqrt{\left(\eta_{q}-\eta_{j}\right)^{2}+\left(\phi_{q}-\phi_{j}\right)^{2}} ; \eta_{q}, \eta_{j}$ are pseudorapidities and $\phi_{q}, \phi_{j}$ are azimuthal angles of $b$-quark and jet respectively. If both of the subjets are found to be $b$-like satisfying matching criteria, then it is 
TABLE I. Parameters, BRs, Higgsino components $\left(N_{13}^{2}, N_{14}^{2}\right)$ of the singlino and fraction of annihilation channels contributing to relic density corresponding to few benchmark points(BP). Energy units are in GeV.

\begin{tabular}{lcccccc}
\hline \hline & BP1 & BP2 & BP3 & BP4 & BP5 & BP6 \\
\hline$\lambda$ & 0.34195 & 0.17783 & 0.22140 & 0.24670 & 0.24980 & 0.29853 \\
$\kappa$ & 0.00080 & 0.00241 & -0.00564 & 0.00520 & -0.00690 & 0.00438 \\
$\tan \beta$ & 8.46 & 5.99 & 4.79 & 5.85 & 4.96 & 4.63 \\
$A_{\lambda}$ & 3114.53 & 793.52 & 1201.50 & 1654.39 & 1968.95 & 1528.60 \\
$A_{\kappa}$ & -46.48 & -29.91 & 36.66 & -57.21 & 69.65 & -60.15 \\
$\mu_{\text {eff }}$ & 340.39 & 150.68 & 232.94 & 290.40 & 378.55 & 364.86 \\
$m_{H_{2}}$ & 123 & 126 & 126 & 126 & 123 & 127 \\
$m_{H_{1}}$ & 43 & 14 & 28 & 36 & 44 & 56 \\
$m_{A_{1}}$ & 8 & 12 & 24 & 31 & 47 & 30 \\
$m_{\tilde{\chi}_{1}^{0}}$ & 3 & 5 & 10 & 14 & 20 & 13 \\
$N_{13}^{2}$ & $10^{-4}$ & $4 \times 10^{-7}$ & $3 \times 10^{-4}$ & $10^{-6}$ & $3 \times 10^{-4}$ & $10^{-4}$ \\
$N_{14}^{2}$ & 0.03 & 0.04 & 0.02 & 0.02 & 0.01 & 0.02 \\
$\Omega h^{2}$ & 0.1115 & 0.1188 & 0.1188 & 0.1255 & 0.1180 & 0.1098 \\
$\operatorname{BR}\left(H_{2} \rightarrow H_{1} H_{1}\right)$ & 0.0001 & 0.06 & 0.01 & 0.11 & 0.08 & 0.07 \\
$\operatorname{BR}\left(H_{2} \rightarrow A_{1} A_{1}\right)$ & 0.10 & 0.004 & 0.06 & 0.001 & 0.02 & 0.01 \\
$\operatorname{BR}\left(H_{1} \rightarrow b \bar{b}\right)$ & 0.81 & 0.57 & 0.75 & 0.22 & 0.50 & 0.50 \\
$\operatorname{BR}\left(H_{1} \rightarrow \tilde{\chi}_{1}^{0} \tilde{\chi}_{1}^{0}\right)$ & 0.07 & 0.31 & 0.18 & 0.75 & 0.45 & 0.44 \\
$\operatorname{BR}\left(H_{1} \rightarrow \tau \tau\right)$ & 0.07 & 0.08 & 0.06 & 0.02 & 0.04 & 0.05 \\
$\operatorname{BR}\left(A_{1} \rightarrow b \bar{b}\right)$ & - & 0.35 & 0.32 & 0.55 & 0.18 & 0.73 \\
$\operatorname{BR}\left(A_{1} \rightarrow \tilde{\chi}_{1}^{0} \tilde{\chi}_{1}^{0}\right)$ & 0.22 & 0.13 & 0.64 & 0.40 & 0.80 & 0.19 \\
$\operatorname{BR}\left(A_{1} \rightarrow \tau \tau\right)$ & 0.69 & 0.42 & 0.03 & 0.05 & 0.01 & 0.06 \\
$\operatorname{Annihilation~channels~}$ & $0.90(\tau \tau)$ & $0.90(b \bar{b})$ & $0.92(b \bar{b})$ & $0.92(b \bar{b})$ & $0.91(b \bar{b})$ & $0.92(b \bar{b})$ \\
& $0.09(\mathrm{gg})$ & $0.07(\tau \tau)$ & $0.07(\tau \tau)$ & $0.07(\tau \tau)$ & $0.08(\tau \tau)$ & $0.07(\tau \tau)$ \\
& & $0.02(\mathrm{gg})$ & $0.01(c \bar{c})$ & & & \\
\hline \hline
\end{tabular}

claimed to be tagged as the $\mathrm{HJ}\left(J_{b \bar{b}}\right)$. We found that the tagging efficiency of $J_{b \bar{b}}$ is around $30 \%$ for the lower range of light Higgs boson mass and goes down to around $15 \%$ for higher mass range. The mass of $J_{b \bar{b}}$ is depicted in Fig. 5 for three samples of Higgs bosons masses. Clearly, the mass peaks are observed at the given input masses. However, peaks are observed to be broader for higher Higgs boson masses. In the same figure, the corresponding distributions from backgrounds are also shown, which are not showing clearly any peaks, as expected. Notably, the presence of $J_{b \bar{b}}$ with a peak in its mass distribution is the characteristics of our signal events.

(iii) Nontagged jets: After tagging $J_{b \bar{b}}$, nontagged QCD jets are constructed out of remaining hadrons in the events using anti- $k_{T}$ [91] algorithm with a jet size parameter $R=0.5$. The reconstructed jets are selected with $p_{T}^{j}>20 \mathrm{GeV}$ and $|\eta|<4.0$.

(iv) Missing transverse momentum $\left(E_{T}\right)$ : The missing transverse momentum is constructed by vector addition of momenta of all visible particles, i.e., $\overrightarrow{p_{T}}=-\sum \overrightarrow{p_{T}^{i}}$, where i runs over all constructed collection from the detector. DELPHES stores $\not_{T}$ of each events taking into account detector effects.

\section{A. Signal for low mass of $H_{1} / A_{1}$}

In this subsection, we discuss the search strategy of the signal process, Eq. (4.3), which is very challenging since the masses of intermediate Higgs bosons are too low to have energetic decay products. The decay mode of Higgs bosons to a pair of $\tau$ leptons is preferred over the $b \bar{b}$ in order to avoid huge QCD background, decay channel and for the same reason, the hadronic mode of tau leptons leading to $\tau$-jets are not considered. Hence, in this scenario, we focus on the final state following Eq. (4.3) as,

$$
\ell^{+} \ell^{-}+\mathscr{E}_{T}^{+} \geq 1 \text { jet. }
$$

Note that the combined BR for both the $\tau$ leptons decaying leptonically is very small $(\sim 12 \%)$. Moreover, leptons are too soft with a very low $p_{T} \sim \frac{m_{H_{1} / A_{1}}}{2}$. In this case, dominating sources of SM backgrounds are due to the inclusive Drell-Yan, $t \bar{t}$, and electroweak processes $W+$ jets, $W W+$ jets, $W Z+$ jets. Performing a naive simulation for both signal and background, we try to find the signal sensitivity. For all background processes except $t \bar{t}$, matrix elements are generated in MadGraph5-aMC@NLO-2.6.4 (MG5NLO), then showering and hadronization are performed using PYTHIA8 as before. The $t \bar{t}$ events are fully 
generated using PYTHIA8. In the simulation, leptons(both $e$ and $\mu)$ are selected with $p_{T}^{\ell} \geq 10 \mathrm{GeV}$ and $|\eta|<2.5$. $^{3}$ Requirement of isolated leptons reduces the signal event significantly. The two leptons originating from $\tau$ pairs are not expected to be widely separated. In our simulation, we ensure isolated leptons by checking e-flow objects of DELPHES using following criteria as,

$$
\frac{\sum p_{T}^{R<0.2}}{p_{T, \ell}}<0.1, \quad \ell=e, \mu
$$

where $p_{T}^{R<0.2}$ is the sum of the transverse momentum of all particles which are within $\Delta R<0.2$ with respect to lepton momentum direction. It also ensures that both the signal leptons are separated by $\Delta R>0.2$. Construction of $E_{T}$ and jets (including $b$-jets) are the same as before and performed by DELPHES.

\section{RESULTS AND DISCUSSION}

Identifying various distinguishing features of the signal process, we impose a few event selection cuts to eliminate backgrounds. For example, the characteristics of $J_{b \bar{b}}$ mass $\left(m_{J_{b \bar{b}}}\right)$ distribution, as shown in Fig. 5, are very different for backgrounds and signal events. Therefore, a background rejection cut setting as,

$$
\begin{aligned}
m_{J_{b \bar{b}}} & <30 \mathrm{GeV} \text { for lower mass range, } \\
30 & <m_{J_{b \bar{b}}}<60 \mathrm{GeV} \text { for higher mass range, }
\end{aligned}
$$

is highly effective, in particular, for eliminating $t \bar{t}$ background by $70 \%-80 \%$.

Evidently, the transverse mass between $J_{b \bar{b}}$ and $E_{T}$ is restricted by the SM Higgs boson mass in signal, as shown in Fig. 6 (left), which is not the case for backgrounds. Hence an upper cut on it as,

$$
\begin{aligned}
& m_{T}\left(J_{b \bar{b}}, \mathscr{E}_{T}\right) \\
& =\sqrt{2 \times p_{T}^{J_{b \bar{b}}} \times \mathscr{E}_{T} \times\left(1-\cos \phi\left(J_{b \bar{b}}, \mathscr{E}_{T}\right)\right)}<140 \mathrm{GeV},
\end{aligned}
$$

is found to be helpful in suppressing background. Another interesting observable is useful in reducing the top background, which is defined as[92],

$$
R\left(n_{j}^{\min }\right)=\frac{\sum_{i=1}^{n_{j}^{\min }}\left|\vec{p}_{T}^{j_{i}}\right|}{H_{T}},
$$

where $n_{j}^{\min }$ is the minimum number of jets required in event selection and $H_{T}=\sum_{i=1}^{n_{j}}\left|\vec{p}_{T} j_{i}\right|$. Obviously, by construction $0<R \leq 1$, where $n_{j}^{\min }$ is set equal to 1 for signal event selection.

\footnotetext{
${ }^{3}$ Experimentally lepton trigger of low $p_{T}$ are to be used.
}

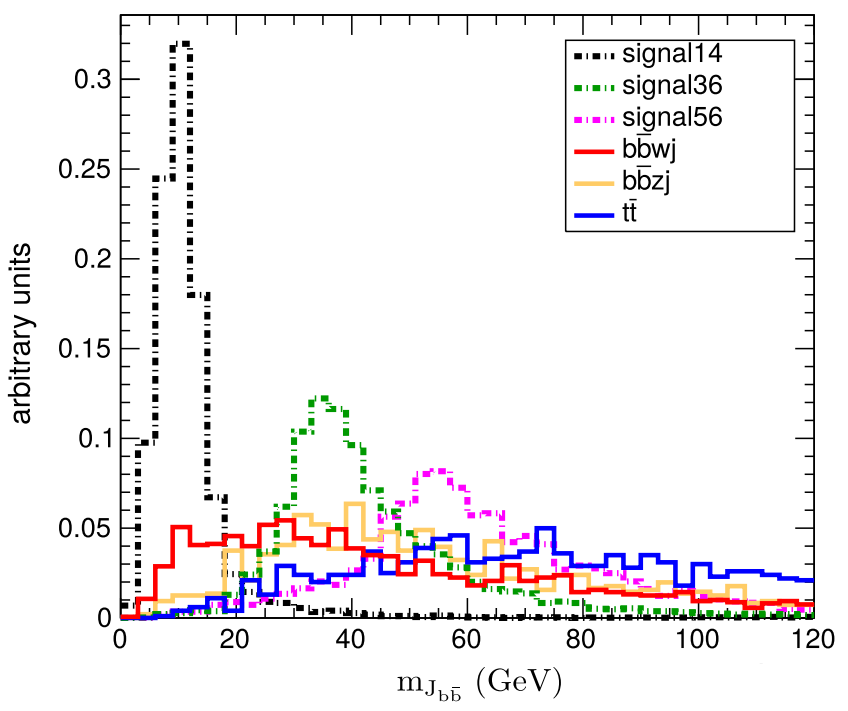

FIG. 5. Mass of $J_{b \bar{b}}$ for three signal points $\left(m_{H_{1}}=14,36\right.$ and $56 \mathrm{GeV})$, and dominant sources of backgrounds.

Distribution of $\mathrm{R}$ is expected to be on the higher side $(R \sim 1)$ for a signal, since it is not very jetty, whereas for $t \bar{t}$ it is expected to be on the lower side, as shown in Fig. 6 (right). Therefore, a selection on $R>0.5$ suppresses a good fraction of top events and to some extent $Z b \bar{b}+$ jets events for moderate mass region.

Cross section yields for the signal which are subject to two different sets of cuts [Eq. (5.1)] on $m_{J_{b \bar{b}}}$ corresponding to benchmark points, and background processes after each set of cuts is presented in Tables II and III.

The first row presents the leading order (LO) cross sections with the center of mass energy $\sqrt{s}=14 \mathrm{TeV}$, setting NNPDF23LO [93] for parton distribution and choosing the dynamic scale $\left(\sqrt{m^{2}+p_{T}^{2}}\right)$ computed by MadGraph5aMC@NLO-2.6.4[83]. Cross sections for the background $\operatorname{processes}(Z b \bar{b}+$ jets, $\quad W b \bar{b}+$ jets $)$ are computed in MadGraph5-aMC@NLO-2.6.4 in five flavor scheme and subject to cuts, $p_{T}^{b}>20 \mathrm{GeV}, p_{T}^{j}>20 \mathrm{GeV}, \Delta R(b, b)>0.1$ and $\Delta R(j, j)>0.4$. Higher order effects to all these cross sections are taken into account through $K$-factors, as defined, $K=\frac{\sigma_{\mathrm{NLO}}}{\sigma_{\mathrm{LO}}}$. These $K$-factors are obtained by computing respective cross sections using MCFM [94-97]. The $K$-factors of the processes, $Z b \bar{b}+$ jets and $W b \bar{b}+$ jets, are considered to be the same as for the processes $Z b \bar{b}$ and $W b \bar{b}$, which are computed by MCFM and found to be $\sim 1.7$ and $\sim 2.6$ respectively, and in close agreement with Ref. [98]. For $t \bar{t}, K$-factor $=1.4$ is used [99,100]. For signal process, $K$-factor is estimated to be $\sim 1.8$ using MCFM, close to quoted values in Ref. [101]. All these $K$-factors are taken into account in Tables II and III while presenting final yields at the end. In these tables, $\epsilon_{\mathrm{BR}}$ is the sum of the branching ratios $\operatorname{BR}\left(H_{2} \rightarrow H_{1} H_{1}\right)$ and $\operatorname{BR}\left(H_{2} \rightarrow A_{1} A_{1}\right)$. 

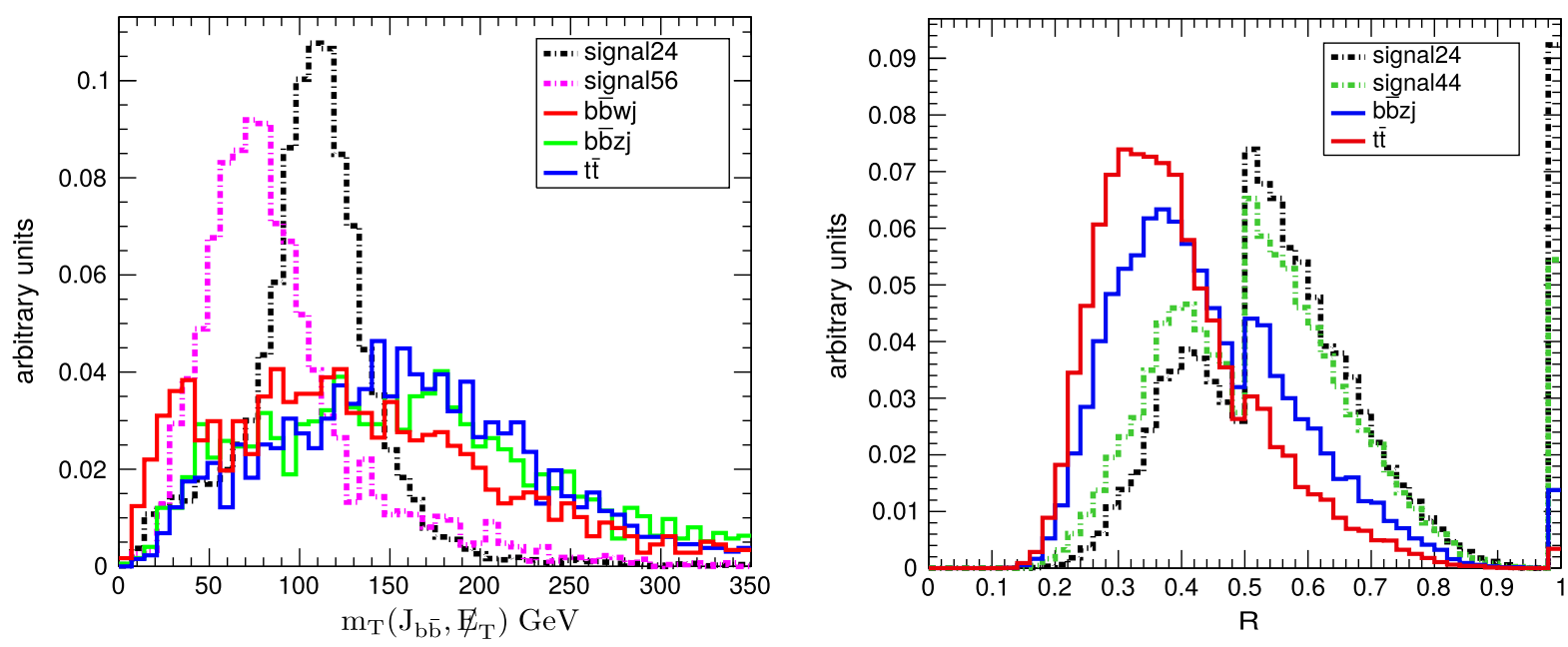

FIG. 6. Transverse mass between $J_{b \bar{b}}$ and $E_{T}$ (Eq. (5.2) (left) and R(Eq. (5.3)(right) for signal (with $m_{A_{1}}=24 \mathrm{GeV}$ and $m_{H_{1}}=56$ (left) or 44 (right) $\mathrm{GeV}$ ), $b \bar{b} Z+$ jets and $t \bar{t}$.

Events are required to contain at least one jet with cuts $p_{T}^{j}>20 \mathrm{GeV}$ and $|\eta|<3$ and vetoed out if there is any lepton. The $E_{T}^{\prime}$ cut is useful in reducing the backgrounds, in particular due to the process with a $\mathrm{Z}$ and $\mathrm{W}$ boson in the final state, however, it costs signal also by almost a factor of 2 , even it is more severe for signal corresponding to lower mass $\sim 15 \mathrm{GeV}$. Notice that the selection of $J_{b \bar{b}}$, and the respective mass window [Eq. (5.1)] suppress backgrounds substantially, by almost two orders of magnitude, while signal remains less affected. A cut on the transverse

TABLE II. Cross section yields after each set of cuts for two low mass signal points BP2 and BP3(Table I) and background processes. Last row presents the final cross sections after including $K$-factor and $b$ tagging efficiency.

\begin{tabular}{lcccrc}
\hline \hline & BP2 & BP3 & $b \bar{b} Z+$ jets & $b \bar{b} W+$ jets & $t \bar{t}$ \\
\hline$\sigma(\mathrm{pb})$ & 12.4 & 12.4 & 152.8 & 139.8 & 597.9 \\
$\sigma \times \epsilon_{\mathrm{BR}}$ & 0.7 & 0.9 & 152.8 & 139.8 & 597.9 \\
lepton veto & 0.6 & 0.8 & 108.5 & 97.6 & 298.2 \\
$n_{j} \geq 1$ & 0.5 & 0.7 & 107.4 & 96.3 & 297.7 \\
$E_{T}>40.0 \mathrm{GeV}$ & 0.3 & 0.4 & 32.8 & 24.4 & 109.4 \\
No. of $J_{b \bar{b}}=1$ & 0.05 & 0.06 & 1.8 & 3.0 & 4.9 \\
$m_{J_{b \bar{b}}}<30.0 \mathrm{GeV}$ & 0.05 & 0.05 & 0.3 & 1.0 & 1.3 \\
$m_{T}\left(J_{b \bar{b}}, E_{T}\right) \leq 140 \mathrm{GeV}$ & 0.04 & 0.04 & 0.2 & 0.8 & 0.9 \\
$R>0.5$ & 0.034 & 0.04 & 0.08 & 0.6 & 0.4 \\
$\sigma \times K$-factor $\times \epsilon_{b}^{2}$ & 0.018 & 0.022 & 0.04 & 0.47 & 0.24 \\
\hline \hline
\end{tabular}

TABLE III. Same as Table II, but for three "high mass" points, BP4, BP5, and BP6) in Table I.

\begin{tabular}{lccccrr}
\hline \hline & BP4 & BP5 & BP6 & $b \bar{b} Z+$ jets & $b \bar{b} W+$ jets & $t \bar{t}$ \\
\hline$\sigma(\mathrm{pb})$ & 12.4 & 12.4 & 12.4 & 152.8 & 139.8 & 597.9 \\
$\sigma \times \epsilon_{\mathrm{BR}}$ & 1.3 & 1.2 & 1.0 & 152.4 & 139.8 & 597.9 \\
Lepton veto & 1.3 & 1.1 & 0.9 & 108.6 & 97.6 & 298.2 \\
$n_{j} \geq 1$ & 1.2 & 1.0 & 0.9 & 108.0 & 97.3 & 297.8 \\
$E_{T}>35.0 \mathrm{GeV}$ & 0.9 & 0.6 & 0.4 & 39.4 & 30.4 & 127.9 \\
No. of $J_{b \bar{b}}=1$ & 0.05 & 0.04 & 0.03 & 3.0 & 2.9 & 7.8 \\
$30.0<m_{J_{b \bar{b}}}<60.0 \mathrm{GeV}$ & 0.03 & 0.03 & 0.01 & 0.6 & 0.8 & 1.8 \\
$m_{T}\left(J_{b \bar{b}}, E_{T}\right) \leq 140 \mathrm{GeV}$ & 0.03 & 0.03 & 0.01 & 0.5 & 0.5 & 1.2 \\
$\mathrm{R}>0.5$ & 0.024 & 0.02 & 0.01 & 0.26 & 0.4 & 0.5 \\
$\sigma \times K$-factor $\times \epsilon_{b}^{2}$ & 0.013 & 0.011 & 0.0055 & 0.13 & 0.3 & 0.3 \\
\hline \hline
\end{tabular}


TABLE IV. Signal significances for benchmark points(BP2-BP6) for two luminosity options.

\begin{tabular}{lccccc}
\hline \hline & BP2 & BP3 & BP4 & BP5 & BP6 \\
\hline$\frac{S}{\sqrt{B}}\left(\mathcal{L}=300 \mathrm{fb}^{-1}\right)$ & 11 & 14 & 8 & 7 & 3.5 \\
$\frac{S}{\sqrt{B}}\left(\mathcal{L}=3000 \mathrm{fb}^{-1}\right)$ & 35 & 44 & 25 & 22 & 11 \\
\hline \hline
\end{tabular}

mass, Eq. (5.2), is very effective in isolating the backgrounds without costing signal events too much, as seen in both the tables. Eventually, as expected, the cut on R suppresses the top background further by about $\sim 50 \%$.

Finally, in order to obtain final cross section yields, we take into account $p_{T}$-dependent $b$-tagging efficiency $\left(\epsilon_{b}\right)$ [102]. For $t \bar{t}$ event, we use $\epsilon_{b}=0.66$, whereas for other cases it is set to $\epsilon_{b}=0.55$. The total background cross section is found to be $750 \mathrm{fb}$ and $730 \mathrm{fb}$ corresponding to two sets of selections as described in Tables II and III respectively. We summarize signal significances, as defined $\frac{S}{\sqrt{B}}$, where $\mathrm{S}$ and $\mathrm{B}$ are the total number of signal and background events, corresponding to five benchmark points in Table IV and for two choices of integrated luminosities $\mathcal{L}=300 \mathrm{fb}^{-1}$ and $3000 \mathrm{fb}^{-1}$. It is to be noted that in background estimation, the contribution due to QCD is not taken into account, where jets and mismeasurement of jets can fake as $b$-jets and $E_{T}^{\prime}$ respectively, which is beyond the scope of this current analysis.

Remarkably, the significances are more than $5 \sigma$ even for lower luminosity option.

In Table $\mathrm{V}$, we present signal cross section yield, presented by Eq. (4.7), corresponding to lower range of Higgs boson masses. Cross sections (LO) shown in the 1st row are computed using MadGraph5-aMC@NLO-2.6.4(MG5NLO) subject to cut $p_{T}^{j}>20 \mathrm{GeV}$, whereas in the subsequent rows, those are presented after each set of selection cuts, as shown. Notice the severe effect of selection cut of invariant mass of lepton pair. Finally, at the last row, we present cross sections, multiplying respective $K$-factors to take care of higher order effects. Similar $K$-factors are used for signal process and $t \bar{t}$ process, whereas for DY process it is taken to be 1.3[103]. For electroweak processes, $W+$ jets, $W W+$ jets, $W Z+$ jets $K$-factors are considered to be 1.42 [104], 1.8 [105] and 2.07 [106] respectively. We find the dominant background contributions are mainly due to the $\mathrm{t} \bar{t}$, DY and $W+$ jets processes. We have also checked the background contribution due to $\Upsilon$ and $J / \psi$ production process, and found to be negligible attributing to comparatively harder $E_{T}$ cut.

The total background cross section are obtained to be $\sim \mathrm{fb}$ and signal significance turns out to be, $\frac{S}{\sqrt{B}} \sim 6(19)$ for integrated luminosity options $300 \mathrm{fb}^{-1}\left(3000 \mathrm{fb}^{-1}\right)$.

\section{SUMMARY}

Various experiments for DM searches have excluded a substantial range of its mass. However, the DM candidate with a very low mass is still a viable option to explain the right relic density of our universe. In this study, we explore the scenario with a light DM candidate in the framework of the NMSSM which is constructed to address the $\mu$-problem of the MSSM by adding one additional singlet Higgs scalar with the two Higgs doublets. In this model, the lightest neutralino, assumed to be a LSP of very low mass, is offered as a DM candidate. The significant presence of singlino component in the lightest neutralino helps to evade constraints on DM-nucleon scattering cross section imposed by several experiments. In this proposed scenario, the DM annihilation takes place primarily via resonant process mediated by singletlike light Higgs bosons, which decay to a pair of fermions in the final state. Thus, the suppressed interaction between singlinolike neutralino and singletlike Higgs scalars is responsible to overcome the stringent constraint due to observed relic abundance. Notably, the light non-SM-like Higgs bosons play a role as a portal between the non-SM and the SM sectors present in the initial and final states of the annihilation process respectively.

A representative numerical scan of model parameters is performed taking into account various existing experimental constraints to identify compatible region corresponding to our proposed DM solution. This naive numerical study indicates that the NMSSM parameters of our interest are of the range, $\kappa \sim 10^{-3}-10^{-2}, \lambda \sim 0.1-0.3,\left|A_{\kappa}\right| \sim 10-100 \mathrm{GeV}$ and $A_{\lambda} \gtrsim 800 \mathrm{GeV}$, which are very close to our speculation based on analytical arguments, as discussed in Secs. II and III. Allowed regions of corresponding parameters are demonstrated for the sake of illustration.

TABLE V. Signal and background events for very "low mass" benchmark point(BP1) in the dilepton scenario.

\begin{tabular}{llccccc}
\hline \hline & BP1 & $t \bar{t}$ & DY + jets & $W+$ jets & $W W+$ jets & $W Z+$ jets \\
\hline$\sigma \times \epsilon_{\mathrm{BR}}(\mathrm{pb})$ & 1.2 & 598 & 4242 & $5 \times 10^{4}$ & 116 & 51 \\
$E_{T}>30 \mathrm{GeV}$ & 0.8 & 371.7 & 314.2 & 10771 & 46.8 & 23.7 \\
$n_{j} \geq 1$ & 0.74 & 371.1 & 301.7 & 10516 & 45.2 & 23.3 \\
$N($ lepton $)=2$ & 0.005 & 15.2 & 16.5 & 0.2 & 1.1 & 0.4 \\
$M_{\ell \ell}<10 \mathrm{GeV}$ & 0.0032 & 0.08 & 0.11 & 0.07 & 0.01 & 0.001 \\
$b$-veto & 0.0032 & 0.024 & 0.11 & 0.07 & 0.01 & 0.001 \\
$\sigma \times K$-factor & 0.006 & 0.034 & 0.14 & 0.1 & 0.02 & 0.002 \\
\hline \hline
\end{tabular}


There are various interesting phenomenological implications at the LHC of the singlinolike DM candidate, which are complimentary to direct searches of it in recoil experiments. For instance, in this current study, we have explored the discovery potential of such low mass DM candidate at the LHC corresponding to its high luminosity options. The DM particle is considered to be produced through SM Higgs production. The SM Higgs boson, produced via the standard dominant gluon-gluon fusion process, decays to a pair of light non-SM-like Higgs bosons. Subsequently, one of the light Higgs bosons decays to a pair of DM particle resulting in missing energy, whereas the other one decays primarily to a pair of, either b quarks or $\tau$ leptons, depending on its mass. In order to make the final state more boosted, we required one extra jet accompanied with SM Higgs boson production. The signal final state is characterized by a $\mathrm{HJ}$, and missing transverse energy accompanied with at least one untagged jet. The HJ is tagged by employing sophisticated MD technique. For the lower range of lighter Higgs boson of mass $<10 \mathrm{GeV}$, we consider its decay to a pair of $\tau$ leptons, which eventually considered to decay in the leptonic channel leading to a final state with two leptons of opposite charge along with missing transverse energy and at least one untagged jet. For the sake of presentation of signal sensitivity, six benchmark points are selected covering all possible mass ranges. Detailed simulation for both the signal and backgrounds are carried out taking into account the detector effects by using DELPHES. Investigating both signal and background event characteristics, we have developed search strategy to suppress background contribution corresponding to a given range of light Higgs boson masses. We found that for medium and higher combination of LSP and light Higgs boson masses, as presented by benchmark points, the sensitivity is more than $5 \sigma$ for an integrated luminosity $\mathcal{L}=300 \mathrm{fb}^{-1}$, and for high integrated luminosity option, $\mathcal{L}=3000 \mathrm{fb}^{-1}$, the it further goes up. This study clearly indicates that the discovery potential for most of the mass range which are consistent with DM solution is very promising with a reasonably high luminosity option of the LHC. We have also carried out a simulation for lower mass range of non-SM Higgs boson, less than $10 \mathrm{GeV}$, of Higgs boson in leptonic final states. Our naive study shows a interesting results of achieving signal sensitivity with a reasonable significance. It is to be noted that in this study the uncertainty due to systematics are not considered, which is beyond the scope of the present study. We conclude that the singlinolike LSP may be a very good viable candidate for DM corresponding to its lower mass range, and its signature at the LHC is also robust with a reasonably promising discovery potential for future luminosity options. However nonobservation of any signal event necessarily does not rule out completely the NMSSM with low mass singlino LSP scenario, instead it constrains the combination of cross sections and related branching ratios. One requires a more detailed and exhaustive scan in order to conclude about the complete exclusion of this scenario.

\section{ACKNOWLEDGMENTS}

One of the authors, A. R, wants to give special thanks to Aravind H. Vijay, Suman Chatterjee, Soham Bhattacharya, and Saikat Karmakar for very useful discussions and valuable suggestions.

\section{APPENDIX A: DM ANNIHILATION CROSS SECTION}

\section{Annihilation through s-channel scalar light Higgs}

The cross section for the process $\tilde{\chi}_{1}^{0} \tilde{\chi}_{1}^{0} \rightarrow H_{1} \rightarrow f \bar{f}$ is given by[107,108]

$$
\begin{aligned}
\sigma_{f \bar{f}}^{H_{1}} & =\frac{\omega_{f \bar{f}}^{H_{1}}(s)}{s^{1 / 2} p_{1}(s)}, \\
\omega_{f \bar{f}}^{H_{1}} & =\frac{g_{f \bar{f} H_{1}}^{2} g_{\tilde{\chi}_{0}^{0} \tilde{\chi}_{1}^{0} H_{1}}^{2}}{\left(s-m_{H_{1}}^{2}\right)^{2}+m_{H_{1}}^{2} \Gamma_{H_{1}}^{2}} \frac{\left(s-4 m_{\tilde{\chi}_{1}^{0}}^{2}\right)\left(s-4 m_{f}^{2}\right)}{16 \pi} \sqrt{1-\frac{4 m_{f}^{2}}{s},}
\end{aligned}
$$

Where, $m_{H_{1}}$ and $\Gamma_{H_{1}}$ are mass and decay width of $H_{1}$ respectively; $p_{1}(s)=p_{2}(s)=\frac{1}{2} \sqrt{s-4 m_{\tilde{\chi}_{1}^{0}}^{2}}$ is the magnitude of 3-momentum of the incoming DM particles in CM frame. $g_{f \bar{f} H_{1}}, g_{\tilde{\chi}_{1}^{0} \tilde{\chi}_{1}^{0} H_{1}}$ are $f \bar{f} H_{1}$ and $\tilde{\chi}_{1}^{0} \tilde{\chi}_{1}^{0} H_{1}$ couplings, respectively. The coupling $g_{\tilde{\chi}_{1}^{0} \tilde{\chi}_{1}^{0} H_{1}}$ is given in Eq. (2.10), and $g_{f \bar{f} H_{1}}$ can be written as [28],

$g_{t \bar{t} H_{1} / c \bar{c} H_{1}}=-\frac{m_{t / c} S_{12}}{\sqrt{2} v \sin \beta}, \quad g_{b \bar{b} H_{1} / \tau \tau H_{1}}=\frac{m_{b / \tau} S_{11}}{\sqrt{2} v \cos \beta}$,

with $S_{i j}$ defined by Eq. (2.2), $m_{f}$ is the mass of fermion $f$.

\section{DM annihilation through s-channel pseudoscalar light Higgs}

Using similar notations, only replacing $H_{1}$ by $A_{1}$ (light pseudoscalar Higgs), we have the squared amplitude given by $[107,108]$ :

$$
\begin{aligned}
\sigma_{f \bar{f}}^{A_{1}} & =\frac{\omega_{f \bar{f}}^{A_{1}}(s)}{s^{1 / 2} p_{1}(s)}, \\
\omega_{f \bar{f}}^{A_{1}} & =\frac{g_{f \bar{f} A_{1}}^{2} g_{\tilde{\chi}_{1}^{0} \tilde{\chi}_{1}^{0} A_{1}}^{2}}{\left(s-m_{A_{1}}^{2}\right)^{2}+m_{A_{1}}^{2} \Gamma_{A_{1}}^{2}} \frac{s^{2}}{16 \pi} \sqrt{1-\frac{4 m_{f}^{2}}{s}} .
\end{aligned}
$$

The coupling $g_{\tilde{\chi}_{1}^{0} \tilde{\chi}_{1}^{0} A_{1}}$ is given in Eq. (2.11), and $g_{f \bar{f} A_{1}}$ has similar structure as Eq. (A2) except components of 
pseudoscalar mass matrix $P_{i j}$ [Eq. (2.4)[, replacing $S_{i j}$. Then the "thermally averaged pair-annihilation cross section times velocity", $\langle\sigma v\rangle$, can be obtained as [107]

$$
\langle\sigma v\rangle=\left[\frac{1}{m_{\tilde{\chi}_{1}^{0}}^{2}}\left(1-\frac{3 T}{m_{\tilde{\chi}_{1}^{0}}^{2}}\right) \omega(s)\right]_{s \rightarrow 4 m_{\tilde{\chi}_{1}^{0}}^{2}+6 m_{\tilde{\chi}_{1}^{2}}^{2} T}+O\left(T^{2}\right),
$$

Where $\omega(s)$ is $\omega_{f \bar{f}}^{H_{1}}(s)$ or $\omega_{f \bar{f}}^{A_{1}}(s)$ and $T$ is temperature.

$$
\mathcal{L}=\lambda_{N} \bar{\chi} \chi \bar{\psi} \psi+i \kappa_{1} \bar{\chi} \chi \bar{\psi} \gamma \gamma_{5} \psi+i \kappa_{2} \bar{\chi} \gamma_{5} \chi \bar{\psi} \gamma+\kappa_{3} \bar{\chi} \gamma_{5} \chi \bar{\psi} \gamma_{5} \psi+\kappa_{4} \bar{\chi} \gamma_{\mu} \gamma_{5} \chi \bar{\psi} \bar{\gamma} \gamma_{\mu} \psi+\zeta_{N} \bar{\chi} \gamma_{\mu} \gamma_{5} \chi \bar{\psi} \gamma^{\mu} \gamma_{5} \psi
$$

It can be shown that, in the zero momentum transfer limit, $\bar{u} \gamma_{5} u$ (u can be $\chi$ or $\psi$ ) vanishes and also the time component of $\bar{u} \gamma_{\mu} \gamma_{5} u$ and the space component of $\bar{u} \gamma_{\mu} u$ tends to zero.

\section{SI cross section}

So the effective spin independent interaction can be written as:

$$
\mathcal{L}^{\mathrm{SI}}=\lambda_{N} \bar{\chi} \chi \bar{\psi} \psi
$$

In our case, this spin-independent scattering cross section $\left(\sigma_{S I}\right)$ of $\tilde{\chi}_{1}^{0}$ with nuclei dominantly happens through exchange of scalar Higgs bosons. When $\tilde{\chi}_{1}^{0}$ is singlinolike, we can write the scattering cross section approximately as [38]

$$
\sigma_{\mathrm{SI}} \simeq \frac{1}{\pi m_{H_{1}}^{4}}\left(\frac{m_{p} m_{\tilde{\chi}_{1}^{0}}}{m_{p}+m_{\tilde{\chi}_{1}^{0}}}\right)^{2} g_{\tilde{\chi}_{1}^{0} \tilde{\chi}_{1}^{0} H_{1}}^{2}\left(\sum_{q=d, s, b} \frac{m_{q} S_{11}}{\cos \beta}\langle N|q \bar{q}| N\rangle+\sum_{q=u, c} \frac{m_{q} S_{12}}{\sin \beta}\langle N|q \bar{q}| N\rangle\right)^{2}
$$

Where, $\langle N|q \bar{q}| N\rangle$ are the matrix element over the atomic nuclear states, $m_{p}$ is the mass of the nuclei. Other notations have usual meanings.

\section{SD cross section}

The effective lagrangian in this case can be written as:

$$
\zeta_{N} \bar{\chi} \gamma_{\mu} \gamma_{5} \chi \bar{\psi} \gamma^{\mu} \gamma_{5} \psi
$$

Here DM-nucleon scattering can be mediated in t-channel by Z-boson or squark mediator (I denote it as V, with mass $m_{V}$ ). The cross section in this case becomes:

$$
\sigma_{\mathrm{SD}} \simeq \frac{4}{\pi m_{V}^{4}}\left(\frac{m_{p} m_{\tilde{\chi}_{1}^{0}}}{m_{p}+m_{\tilde{\chi}_{1}^{0}}}\right)^{2}\left(\frac{J_{A}+1}{J_{A}}\right) g_{\tilde{\chi}_{1}^{0} \tilde{\chi}_{1}^{0} V}^{2}\left(\sum_{q=u, d, c, s, b}\langle N|q \bar{q}| N\rangle\right)^{2}\left(\zeta_{p} S_{p}^{A}+\zeta_{n} S_{n}^{A}\right)^{2}
$$

where $J_{A}$ is the angular momentum of the nucleus with A nucleons and $S_{N}^{A}$ are the expectation value of the spin content of nucleon type $N(n$ or $p)$.

[1] D. Hooper and E. A. Baltz, Strategies for determining the nature of dark matter, Annu. Rev. Nucl. Part. Sci. 58, 293 (2008).
[2] J. L. Feng, Dark matter candidates from particle physics and methods of detection, Annu. Rev. Astron. Astrophys. 48, 495 (2010). 
[3] N. Aghanim et al. (Planck Collaboration), Planck 2018 results. VI. Cosmological parameters, Astron. Astrophys. 641, A6 (2020).

[4] E. Aprile et al. (XENON Collaboration), Dark Matter Search Results from a One Ton-Year Exposure of XENON1T, Phys. Rev. Lett. 121, 111302 (2018).

[5] D. S. Akerib et al. (LUX Collaboration), Results from a Search for Dark Matter in the Complete LUX Exposure, Phys. Rev. Lett. 118, 021303 (2017).

[6] X. Cui et al. (PandaX-II Collaboration), Dark Matter Results From 54-Ton-Day Exposure of PandaX-II Experiment, Phys. Rev. Lett. 119, 181302 (2017).

[7] P. Agnes et al. (DarkSide Collaboration), Low-Mass Dark Matter Search with the DarkSide-50 Experiment, Phys. Rev. Lett. 121, 081307 (2018).

[8] E. Aprile et al. (XENON Collaboration), Constraining the Spin-Dependent WIMP-Nucleon Cross Sections with XENON1T, Phys. Rev. Lett. 122, 141301 (2019).

[9] C. Amole et al. (PICO Collaboration), Dark matter search results from the complete exposure of the PICO- $60 \mathrm{C}_{3} \mathrm{~F}_{8}$ bubble chamber, Phys. Rev. D 100, 022001 (2019).

[10] V. Barger, W.-Y. Keung, and G. Shaughnessy, Spin dependence of dark matter scattering, Phys. Rev. D 78, 056007 (2008).

[11] G. Belanger, F. Boudjema, A. Pukhov, and A. Semenov, Dark matter direct detection rate in a generic model with micromegas 2.2, Comput. Phys. Commun. 180, 747 (2009).

[12] P. Agrawal, Z. Chacko, C. Kilic, and R. K. Mishra, A classification of dark matter candidates with primarily spin-dependent interactions with matter, arXiv:1003.1912.

[13] C. E. Aalseth et al. (CoGeNT Collaboration), Results from a Search for Light-Mass Dark Matter with a P-type Point Contact Germanium Detector, Phys. Rev. Lett. 106, 131301 (2011).

[14] R. Agnese et al. (CDMS Collaboration), Silicon Detector Dark Matter Results from the Final Exposure of CDMS II, Phys. Rev. Lett. 111, 251301 (2013).

[15] R. Bernabei et al., Final model independent result of DAMA/LIBRA-phase1, Eur. Phys. J. C 73, 2648 (2013).

[16] D. Abercrombie et al., Dark matter benchmark models for early LHC run-2 searches: Report of the ATLAS/CMS dark matter forum, Phys. Dark Universe 26, 100371 (2019).

[17] N. Trevisani (ATLAS, CMS Collaborations), Collider searches for dark matter (ATLAS + CMS), Universe 4, 131 (2018).

[18] M. Aaboud et al. (ATLAS Collaboration), Constraints on mediator-based dark matter and scalar dark energy models using $\sqrt{s}=13 \mathrm{TeV} p p$ collision data collected by the ATLAS detector, J. High Energy Phys. 05 (2019) 142.

[19] G. Bertone, D. Hooper, and J. Silk, Particle dark matter: Evidence, candidates and constraints, Phys. Rep. 405, 279 (2005).

[20] G. Belanger, Dark matter and the LHC, Nucl. Phys. B, Proc. Suppl. 194, 5 (2009).

[21] N. Arkani-Hamed, A. Delgado, and G. Giudice, The welltempered neutralino, Nucl. Phys. B741, 108 (2006).

[22] H. Baer, V. Barger, and H. Serce, SUSY under siege from direct and indirect WIMP detection experiments, Phys. Rev. D 94, 115019 (2016).
[23] M. Chakraborti, U. Chattopadhyay, and S. Poddar, How light a Higgsino or a wino dark matter can become in a compressed scenario of MSSM, J. High Energy Phys. 09 (2017) 064.

[24] R. K. Barman, G. Belanger, B. Bhattacherjee, R. Godbole, G. Mendiratta, and D. Sengupta, Invisible decay of the Higgs boson in the context of a thermal and nonthermal relic in MSSM, Phys. Rev. D 95, 095018 (2017).

[25] P. Fayet, Supergauge invariant extension of the Higgs mechanism and a model for the electron and its neutrino, Nucl. Phys. B90, 104 (1975).

[26] M. Drees, Supersymmetric models with extended higgs sector, Int. J. Mod. Phys. A 04, 3635 (1989).

[27] J. R. Ellis, J. F. Gunion, H. E. Haber, L. Roszkowski, and F. Zwirner, Higgs bosons in a nonminimal supersymmetric model, Phys. Rev. D 39, 844 (1989).

[28] U. Ellwanger, C. Hugonie, and A. M. Teixeira, The nextto-minimal supersymmetric standard model, Phys. Rep. 496, 1 (2010).

[29] J. E. Kim and H. Nilles, The $\mu$-problem and the strong $C P$ problem, Phys. Lett. B 138, 150 (1984).

[30] U. Ellwanger, Higgs bosons in the next-to-minimal supersymmetric standard model at the LHC, Eur. Phys. J. C 71, 1782 (2011).

[31] N. D. Christensen, T. Han, Z. Liu, and S. Su, Low-mass Higgs bosons in the NMSSM and their LHC implications, J. High Energy Phys. 08 (2013) 019.

[32] J. Cao, F. Ding, C. Han, J. M. Yang, and J. Zhu, A light Higgs scalar in the NMSSM confronted with the latest LHC Higgs data, J. High Energy Phys. 11 (2013) 018.

[33] M. Guchait and J. Kumar, Light Higgs bosons in NMSSM at the LHC, Int. J. Mod. Phys. A 31, 1650069 (2016).

[34] F. Domingo and G. Weiglein, NMSSM interpretations of the observed Higgs signal, J. High Energy Phys. 04 (2016) 095.

[35] J. Kumar and M. Paraskevas, Distinguishing between MSSM and NMSSM through $\Delta F=2$ processes, J. High Energy Phys. 10 (2016) 134.

[36] M. Guchait and J. Kumar, Diphoton signal of a light pseudoscalar in the NMSSM at the LHC, Phys. Rev. D 95, 035036 (2017).

[37] G. Belanger, F. Boudjema, C. Hugonie, A. Pukhov, and A. Semenov, Relic density of dark matter in the NMSSM, J. Cosmol. Astropart. Phys. 09 (2005) 001.

[38] J. F. Gunion, D. Hooper, and B. McElrath, Light neutralino dark matter in the NMSSM, Phys. Rev. D 73, 015011 (2006).

[39] C. Hugonie, G. Belanger, and A. Pukhov, Dark matter in the constrained NMSSM, J. Cosmol. Astropart. Phys. 11 (2007) 009.

[40] U. Ellwanger and A. M. Teixeira, NMSSM with a singlino LSP: Possible challenges for searches for supersymmetry at the LHC, J. High Energy Phys. 10 (2014) 113.

[41] U. Ellwanger, Present status and future tests of the Higgsino-singlino sector in the NMSSM, J. High Energy Phys. 02 (2017) 051.

[42] Q. Mou and S. Zheng, Light neutralino dark matter in the NMSSM, arXiv:1703.00343. 
[43] U. Ellwanger and C. Hugonie, The Higgsino-singlino sector of the NMSSM: Combined constraints from dark matter and the LHC, Eur. Phys. J. C 78, 735 (2018).

[44] W. Abdallah, A. Chatterjee, and A. Datta, Revisiting singlino dark matter of the natural $Z_{3}$-symmetric NMSSM in the light of LHC, J. High Energy Phys. 09 (2019) 095.

[45] S. Baum, M. Carena, N. R. Shah, and C. E. Wagner, Higgs portals for thermal Dark Matter. EFT perspectives and the NMSSM, J. High Energy Phys. 04 (2018) 069.

[46] G. Arcadi, A. Djouadi, and M. Raidal, Dark matter through the Higgs portal, Phys. Rep. 842, 1 (2020).

[47] J. Huang, T. Liu, L.-T. Wang, and F. Yu, Supersymmetric Exotic Decays of the $125 \mathrm{GeV}$ Higgs Boson, Phys. Rev. Lett. 112, 221803 (2014).

[48] J. Huang, T. Liu, L.-T. Wang, and F. Yu, Supersymmetric subelectroweak scale dark matter, the Galactic Center gamma-ray excess, and exotic decays of the $125 \mathrm{GeV}$ Higgs boson, Phys. Rev. D 90, 115006 (2014).

[49] C. T. Potter, Natural NMSSM with a light singlet Higgs and singlino LSP, Eur. Phys. J. C 76, 44 (2016).

[50] Q.-F. Xiang, X.-J. Bi, P.-F. Yin, and Z.-H. Yu, Searching for singlino-Higgsino dark matter in the NMSSM, Phys. Rev. D 94, 055031 (2016).

[51] M. Guchait and J. Kumar, Light Higgs bosons in NMSSM at the LHC, Int. J. Mod. Phys. A 31, 1650069 (2016).

[52] M. Guchait, A. H. Vijay, and J. Kumar, Detection prospects of light pseudoscalar Higgs boson at the LHC, J. High Energy Phys. 08 (2017) 122.

[53] J. R. Andersen et al. (LHC Higgs Cross Section Working Group Collaboration), Handbook of LHC Higgs cross sections: 3. Higgs properties, arXiv:1307.1347.

[54] S. Dittmaier et al. (LHC Higgs Cross Section Working Group Collaboration), Handbook of LHC higgs cross sections: 1. Inclusive observables, arXiv:1101.0593.

[55] J. M. Butterworth, A. R. Davison, M. Rubin, and G. P. Salam, Jet Substructure as a New Higgs Search Channel at the LHC, Phys. Rev. Lett. 100, 242001 (2008).

[56] R. Kogler et al., Jet substructure at the large hadron collider: Experimental review, Rev. Mod. Phys. 91, 045003 (2019).

[57] D. J. Miller, R. Nevzorov, and P. M. Zerwas, The Higgs sector of the next-to-minimal supersymmetric standard model, Nucl. Phys. B681, 3 (2004).

[58] LEPSUSYWG, Aleph, Delphi, L3 and OPAL experiments, note LEPSUSYWG/01-03.1, Combined LEP chargino mass limits, http://lepsusy.web.cern.ch/lepsusy.

[59] T. Moroi and Y. Okada, Upper bound of the lightest neutral Higgs mass in extended supersymmetric standard models, Phys. Lett. B 295, 73 (1992).

[60] F. Franke and H. Fraas, Neutralinos and Higgs bosons in the next-to-minimal supersymmetric standard model, Int. J. Mod. Phys. A 12, 479 (1997).

[61] S. F. King, M. Muhlleitner, and R. Nevzorov, NMSSM Higgs benchmarks near $125 \mathrm{GeV}$, Nucl. Phys. B860, 207 (2012).

[62] K. Agashe, Y. Cui, and R. Franceschini, Natural Islands for a $125 \mathrm{GeV}$ Higgs in the scale-invariant NMSSM, J. High Energy Phys. 02 (2013) 031.

[63] D. Albornoz Vasquez, G. Belanger, C. Boehm, J. Da Silva, P. Richardson, and C. Wymant, The $125 \mathrm{GeV}$ Higgs in the
NMSSM in light of LHC results and astrophysics constraints, Phys. Rev. D 86, 035023 (2012).

[64] M. Badziak, M. Olechowski, and S. Pokorski, New regions in the NMSSM with a $125 \mathrm{GeV}$ Higgs, J. High Energy Phys. 06 (2013) 043.

[65] L. J. Hall, D. Pinner, and J. T. Ruderman, A natural SUSY Higgs near 126 GeV, J. High Energy Phys. 04 (2012) 131.

[66] M. Guchait, Exact solution of the neutralino mass matrix, Z. Phys. C 57, 157 (1993); Erratum, Z. Phys. C 61, 178 (1994).

[67] S. Y. Choi, J. Kalinowski, G. A. Moortgat-Pick, and P. M. Zerwas, Analysis of the neutralino system in supersymmetric theories, Eur. Phys. J. C 22, 563 (2001); Eur. Phys. J. C 23, 769(A) (2002).

[68] P. N. Pandita, Approximate formulas for the neutralino masses in the nonminimal supersymmetric standard model, Phys. Rev. D 50, 571 (1994).

[69] S. Y. Choi, D. J. Miller, and P. M. Zerwas, The neutralino sector of the next-to-minimal supersymmetric standard model, Nucl. Phys. B711, 83 (2005).

[70] J. Kozaczuk and S. Profumo, Light NMSSM neutralino dark matter in the wake of CDMS II and a $126 \mathrm{GeV}$ Higgs boson, Phys. Rev. D 89, 095012 (2014).

[71] T. Han, Z. Liu, and S. Su, Light neutralino dark matter: Direct/indirect detection and collider searches, J. High Energy Phys. 08 (2014) 093.

[72] K. Wang and J. Zhu, The funnel annihilations of light dark matter and the Higgs invisible decay, Phys. Rev. D 101, 095028 (2020).

[73] M. Guchait and J. Kumar, Diphoton Signal of light pseudoscalar in NMSSM at the LHC, Phys. Rev. D 95, 035036 (2017).

[74] U. Ellwanger, J. F. Gunion, and C. Hugonie, NMHDECAY: A Fortran code for the Higgs masses, couplings and decay widths in the NMSSM, J. High Energy Phys. 02 (2005) 066.

[75] U. Ellwanger and C. Hugonie, NMHDECAY 2.0: An updated program for sparticle masses, Higgs masses, couplings and decay widths in the NMSSM, Comput. Phys. Commun. 175, 290 (2006).

[76] G. Belanger, F. Boudjema, A. Pukhov, and A. Semenov, micromeGAs: Version 1.3, Comput. Phys. Commun. 174, 577 (2006).

[77] G. Belanger, F. Boudjema, A. Pukhov, and A. Semenov, MicromeGAs 2.0: A program to calculate the relic density of dark matter in a generic model, Comput. Phys. Commun. 176, 367 (2007).

[78] G. Belanger, F. Boudjema, A. Pukhov, and A. Semenov, micrOMEGAs 3: A program for calculating dark matter observables, Comput. Phys. Commun. 185, 960 (2014).

[79] S. Baum, N. R. Shah, and K. Freese, The NMSSM is within reach of the LHC: Mass correlations \& decay signatures, J. High Energy Phys. 04 (2019) 011.

[80] C. Beskidt and W. de Boer, Effective scanning method of the NMSSM parameter space, Phys. Rev. D 100, 055007 (2019).

[81] C. Beskidt and W. de Boer, Genuine NMSSM deviations in the $125 \mathrm{GeV}$ Higgs boson decays,

[82] ATLAS Collaboration, Combined measurements of Higgs boson production and decay using up to $80 \mathrm{fb}^{-1}$ of 
proton-proton collision data at $\sqrt{s}=13 \mathrm{TeV}$ collected with the ATLAS experiment, Technical Report No. ATLASCONF-2018-031, CERN, Geneva, 2018, https://cds.cern .ch/record/2629412.

[83] J. Alwall, R. Frederix, S. Frixione, V. Hirschi, F. Maltoni, O. Mattelaer, H. S. Shao, T. Stelzer, P. Torrielli, and M. Zaro, The automated computation of tree-level and nextto-leading order differential cross sections, and their matching to parton shower simulations, J. High Energy Phys. 07 (2014) 079.

[84] T. Sjostrand, S. Mrenna, and P.Z. Skands, PYTHIA 6.4 physics and manual, J. High Energy Phys. 05 (2006) 026.

[85] T. Sjostrand, S. Mrenna, and P.Z. Skands, A brief introduction to PYTHIA 8.1, Comput. Phys. Commun. 178, 852 (2008).

[86] P. Z. Skands et al., SUSY Les Houches accord: Interfacing SUSY spectrum calculators, decay packages, and event generators, J. High Energy Phys. 07 (2004) 036.

[87] J. de Favereau, C. Delaere, P. Demin, A. Giammanco, V. Lemaître, A. Mertens, and M. Selvaggi (DELPHES 3 Collaboration), DELPHES 3, A modular framework for fast simulation of a generic collider experiment, J. High Energy Phys. 02 (2014) 057.

[88] M. Cacciari, G. P. Salam, and G. Soyez, FastJet user manual, Eur. Phys. J. C 72, 1896 (2012).

[89] Y. L. Dokshitzer, G. D. Leder, S. Moretti, and B. R. Webber, Better jet clustering algorithms, J. High Energy Phys. 08 (1997) 001.

[90] M. Dasgupta, A. Fregoso, S. Marzani, and G. P. Salam, Towards an understanding of jet substructure, J. High Energy Phys. 09 (2013) 029.

[91] M. Cacciari, G. P. Salam, and G. Soyez, The anti- $k_{t}$ jet clustering algorithm, J. High Energy Phys. 04 (2008) 063.

[92] M. Guchait and D. Sengupta, Event-shape selection cuts for supersymmetry searches at the LHC with $7 \mathrm{TeV}$ energy, Phys. Rev. D 84, 055010 (2011).

[93] R. D. Ball, L. Del Debbio, S. Forte, A. Guffanti, J. I. Latorre, J. Rojo, and M. Ubiali, A first unbiased global NLO determination of parton distributions and their uncertainties, Nucl. Phys. B838, 136 (2010).

[94] J. M. Campbell and R. K. Ellis, An update on vector boson pair production at hadron colliders, Phys. Rev. D 60 , 113006 (1999).

[95] J. M. Campbell, R. K. Ellis, and C. Williams, Vector boson pair production at the LHC, J. High Energy Phys. 07 (2011) 018.
[96] J. M. Campbell, R. K. Ellis, and W. T. Giele, A multithreaded version of MCFM, Eur. Phys. J. C 75, 246 (2015).

[97] R. Boughezal, J. M. Campbell, R. K. Ellis, C. Focke, W. Giele, X. Liu, F. Petriello, and C. Williams, Color singlet production at NNLO in MCFM, Eur. Phys. J. C 77, 7 (2017).

[98] F. Febres Cordero, L. Reina, and D. Wackeroth, W- and $Z$-boson production with a massive bottom-quark pair at the Large Hadron Collider, Phys. Rev. D 80, 034015 (2009).

[99] K. Melnikov and M. Schulze, NLO QCD corrections to top quark pair production and decay at hadron colliders, J. High Energy Phys. 08 (2009) 049.

[100] N. Kidonakis and R. Vogt, The theoretical top quark cross section at the tevatron and the LHC, Phys. Rev. D 78, 074005 (2008).

[101] D. de Florian et al. (LHC Higgs Cross Section Working Group Collaboration), Handbook of LHC Higgs cross sections: 4. Deciphering the nature of the Higgs sector, arXiv:1610.07922.

[102] A. M. Sirunyan et al. (CMS Collaboration), Identification of heavy-flavour jets with the CMS detector in pp collisions at $13 \mathrm{TeV}$, J. Instrum. 13, P05011 (2018).

[103] A. H. Ajjath, G. Das, M. Kumar, P. Mukherjee, V. Ravindran, and K. Samanta, Resummed Drell-Yan cross-section at $\mathrm{N}^{3} \mathrm{LL}$, arXiv:2001.11377.

[104] R. Boughezal, C. Focke, X. Liu, and F. Petriello, W-Boson Production in Association with a Jet at Next-to-Next-toLeading Order in Perturbative QCD, Phys. Rev. Lett. 115, 062002 (2015).

[105] T. Gehrmann, M. Grazzini, S. Kallweit, P. Maierhöfer, A. von Manteuffel, S. Pozzorini, D. Rathlev, and L. Tancredi, $W^{+} W^{-}$Production at Hadron Colliders in Next to Next to Leading Order QCD, Phys. Rev. Lett. 113, 212001 (2014).

[106] M. Grazzini, S. Kallweit, D. Rathlev, and M. Wiesemann, $W^{ \pm} Z$ production at hadron colliders in NNLO QCD, Phys. Lett. B 761, 179 (2016).

[107] J. R. Ellis, T. Falk, K. A. Olive, and M. Srednicki, Calculations of neutralino-stau coannihilation channels and the cosmologically relevant region of MSSM parameter space, Astropart. Phys. 13, 181 (2000); Erratum, 15, 413 (2001).

[108] T. Nihei, L. Roszkowski, and R. Ruiz de Austri, Exact cross-sections for the neutralino WIMP pair annihilation, J. High Energy Phys. 03 (2002) 031. 